\title{
Unilateral Vibro-Impact Systems-Experimental Observations against Theoretical Predictions based on the Coefficient of Restitution
}

\author{
de S. Reboucas, Geraldo F.; Santos, IImar; Thomsen, Jon J.
}

Published in:

Journal of Sound and Vibration

Link to article, DOI:

10.1016/j.jsv.2018.10.037

Publication date:

2019

Document Version

Peer reviewed version

Link back to DTU Orbit

Citation (APA):

de S. Reboucas, G. F., Santos, I., \& Thomsen, J. J. (2019). Unilateral Vibro-Impact Systems-Experimental Observations against Theoretical Predictions based on the Coefficient of Restitution. Journal of Sound and Vibration, 440, 346-371. https://doi.org/10.1016/j.jsv.2018.10.037

\section{General rights}

Copyright and moral rights for the publications made accessible in the public portal are retained by the authors and/or other copyright owners and it is a condition of accessing publications that users recognise and abide by the legal requirements associated with these rights.

- Users may download and print one copy of any publication from the public portal for the purpose of private study or research.

- You may not further distribute the material or use it for any profit-making activity or commercial gain

- You may freely distribute the URL identifying the publication in the public portal 


\title{
Unilateral Vibro-Impact Systems - Experimental Observations against Theoretical Predictions based on the Coefficient of Restitution
}

\author{
Geraldo F. de S. Rebouças ${ }^{\mathrm{a}}$, Ilmar F. Santos ${ }^{\mathrm{a}, *}$, Jon J. Thomsen ${ }^{\mathrm{a}}$ \\ ${ }^{a}$ Department of Mechanical Engineering, Technical University of Denmark - DTU, Denmark
}

\begin{abstract}
The vibro-impact response of a single-degree of freedom model with the coefficient of restitution is analyzed using pointwise mapping and a standard averaging combined with non-smooth transformations. Experimental data are taken from a cantilever beam with attached mass and unilateral constraint submitted to different gap configurations and levels of excitation. Numerical simulations are used to reproduce empirical observations to a certain extent and validate theoretical predictions. Investigations on the coefficient of restitution show its dependence on the forcing frequency and pre-contact velocity. The effect of gap variations due to sliding of the constraint during frequency sweep is analyzed experimentally.
\end{abstract}

Keywords: vibro-impact dynamics; unilateral impact; kinematic impact; coefficient of restitution; vibro-impacting beam; beam with attached mass;

\section{Introduction}

Impacts are very common in engineering practice, being necessary for some applications and avoided in others. For instance, while the interaction between different structural components is essential for some processes, like hammering and riveting, the contact between rotor and stator is a problem. Thus, impacts are a widely studied subject in mechanics and applied mathematics.

Roughly speaking, there are two main ways of modeling impacts: using an impact force function or a coefficient of restitution, CoR. Impact forces are very common in literature, with piecewise linear functions of the elastic term being the most common choice [1-10]. Other works regard stiffness and damping as piecewise linear [1114]. Physical inaccuracies on those models motivated the development of formulations where the impact force is a power of both displacement and velocity [15--17], incorporating the $\mathrm{CoR}$ as a parameter. An advantage of using the force approach is that it provides information on structural stresses. However, it also demands knowledge on the contact duration, which can be difficult to obtain.

\footnotetext{
${ }^{*}$ Corresponding author

Email address: if s@mek.dtu.dk (Ilmar F. Santos)
} 
The coefficient of restitution, given by the negative ratio between velocities after and before impact against an immovable stop is another very common way of modeling impacts [18-27], due to its straightforward physical interpretation and formulation. In this approach the impacting elements are considered to be rigid, the contact duration is supposed to be negligible and there is no way to estimate contact forces. While the $\mathrm{CoR}$ is considered a constant parameter in most applications, there is evidence of its dependence on the impact velocity [28].

The impact's nature should be taken into account when choosing the model formulation. For instance, force modeling is more appropriate when contact duration and deformation are not negligible, otherwise, both models should produce similar results, as shown in [29] through numerical simulation.

For problems where impacts are the only nonlinearity, one can use Pointwise mapping [30, 31] to access some model properties, such as stability of solutions and bifurcation points. Other techniques such as Harmonic Linearization [32], Averaging [33], and the Lindstedt-Poincaré method [34] can be used to solve problems with weak smooth and non-smooth nonlinearities, providing information about the stability of solutions and steady-state vibration amplitude. For general impacting systems (not weak), discontinuous transformations [35] of time [36] and state variables [37, 38] can be used to eliminate or weaken non-smooth terms. After that, standard analytical techniques such as Averaging [23, 39] and Multiple Scales [26] can be applied.

Discontinuous transformations can be subdivided according to impact characteristics, such as elastic or inelastic contacts and one or two-sided constraints, also called unilateral/bilateral constraints, respectively. In this context, Zhuravlev developed coordinate transformations to treat problems with unilateral [37] and bilateral [40] purely elastic constraints. Ivanov's transformation [38] can be seen as a generalized version of Zhuravlev's transformation for unilateral problems with dissipative collisions. The non-smooth temporal substitutions proposed by Pilipchuk [35, 36] is applied to bilateral vibro-impact problems with elastic collisions. Recently, this transformation was generalized to inelastic impact problems [41]. For near-elastic vibro-impact problems, Thomsen and Fidlin [23] use Zhuravlev transformation for unilateral constraints and a sawtooth function of the displacement variable for bilateral impacts. Czolczynski et. al. [42] uses a modified Peterka method to analyze the dynamics of a base excited SDOF system, obtaining regions of stability for period- $n$ oscillations and agreement with numerical results.

It is common to use standard ODE solvers together with event handling subroutines to perform numerical simulations of impact vibrations. An alternative is to use smoothed versions of the discontinuities on impact force models directly with common ODE solvers [43-45]. Grace et. al. [46] uses non-smooth coordinate transformations as a preprocessing step to numerical simulations of vibro-impacts systems to weaken the kinematic impact rule. The development of numerical time-integration algorithms for non-smooth models is an active field of research [47-49] whose discussion is out of the scope of this work.

There are many experimental works which use low order models to describe vibroimpacts on continuous structures [2, 3, 11, 13, 18,-21, 50]. Moon and Shaw [2] used a piecewise linear single degree of freedom, SDOF, model to reproduce the chaotic behavior of an experimental base excited cantilever beam with unilateral constraint 
setup, obtaining qualitative agreement. Shaw and Holmes [1] obtain an expression for the stability and bifurcation of periodic orbits in an impacting SDOF model using CoR and piecewise linear impact force, applying pointwise mapping analysis. Those predictions were evaluated by Shaw [3] using an experimental base-excited cantilever beam with unilateral constraint setup, obtaining reasonable quantitative and qualitative agreement.

Stensson and Nordmark [18] use a attached mass cantilever beam driven by intermittent impacts setup to illustrate geometric features particular to attractors of impacting systems. The experiment was modeled with a SDOF model with CoR impact and solved numerically, being able to reproduce the experimental findings. Fang and Wickert [19] model a similar setup using a SDOF model with CoR impact. The system is analyzed using pointwise mapping and numerical simulations. The same modeling was used by Bishop et al. [20] to reproduce the experimental frequency response of a base-excited cantilever beam with a unilateral constraint around its first resonance.

Wagg and Bishop [21] present a technique which enables the usage of many degrees of freedom to model continuous structures with CoR impact, using a base-excited cantilever beam with unilateral constraint setup as a testbed for the presented technique. Andreaus et al. [13] use a 3-DOF piecewise linear model to simulate the experimental behavior of a attached mass harmonically forced cantilever beam with one-sided constraints. The dimensionality of vibro-impact beams has been studied experimentally by [51, 52] using correlation dimension and proper orthogonal decomposition, respectively. Czolczynski et. al. [53], presents numerical comparisons between lowdimensional models (1 and 2 DOFs) against a high-order finite element model of a base-excited cantilever beam with attached mass and unilateral Hertzian impacts.

Experimental bifurcation diagrams for a setup similar to [19] were obtained by Balachandran [50]. The structural impacts were modeled in two ways, with infinitesimal (CoR) and finite contact duration (force). Dick et al. [11], studies grazing bifurcations using a multi-DOF model with piecewise linear contact to model a base-excited cantilever beam with unilateral constraint setup.

There are also many experimental works dealing with unilateral impacts on discrete SDOF systems, e.g. [44, 54,57]. Todd and Virgin [54] used a cart rolling in an energy well track experiment to study the influence of impact location on the cart dynamics. Using a SDOF model with amplitude-dependent natural frequency, they were able to reproduce experimental observations. Savi et al. [44] used a piecewise-linear model to mimic the dynamics of a cart sliding on a straight horizontal rail and hitting a support. As pointed out by [54], those setups eliminate the possibility of modal interactions that can affect the structural impact response. However, the manufacturing of those experiments is more complex, requiring more parts.

The discussion above shows that, under certain conditions, the coefficient of restitution can be used to obtain reasonable agreement between theory and experiments. On the other hand, the motivation and main originality of the present work is to analyse the limitations of the coefficient of restitution model, using experimental observations from a simple vibro-impact setup, investigating the applicability conditions of the CoR model approach. In the present work, this is done through the experimental analysis of a unilateral vibro-impact setup with different gap widths (pre-stressed, neutral and loose) and excitation levels and its subsequent correlation with predictions made in the 


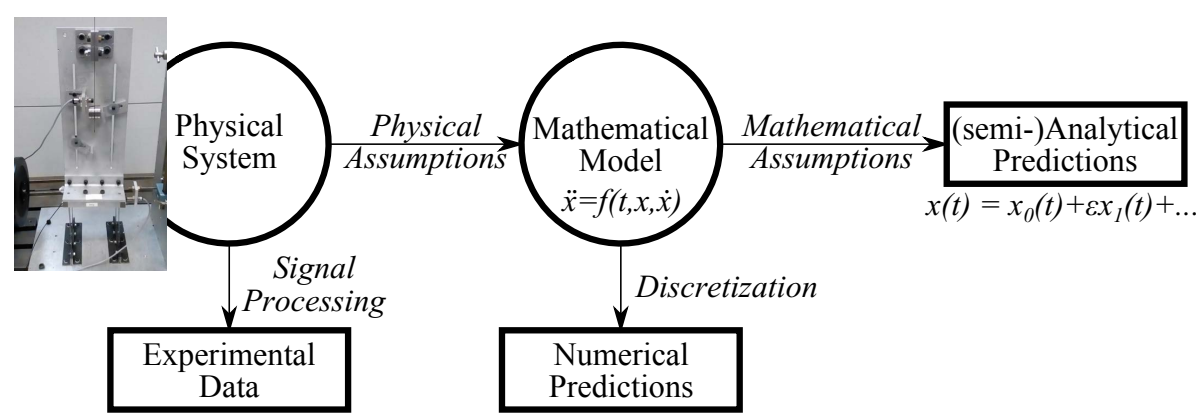

Figure 1: Modeling diagram.

literature using numerical and analytical tools. The effect on the magnitude response of gap deviation during an experimental frequency sweep is discussed. Limitations on the excitation source are discussed. Agreement between theory and experiments is obtained under certain circumstances despite experimental challenges. Usage of discontinuous transformations as a preprocessing step for numerical simulation of vibroimpact problems is considered.

The manuscript is organized as follows: Section 2 discusses the model for a SDOF base-excited mechanical oscillator under single-sided impacts described by a coefficient of restitution. The numerical simulation of this model is discussed in Section 3 , where the computation time is reduced by using non-smooth coordinate transformations as a preprocessing step. Then, standard averaging and pointwise mapping are employed in Section 4 to obtain approximated period-one solutions and investigate their stability. This section concludes with a comparison between the results from numerical and analytical techniques. A brief description of the experimental setup and its mathematical model are presented in Sections 5 and 6 respectively. Numerical and analytical predictions are compared with empirical data in Section 7 Finally, conclusions and future perspectives are presented in Section 8.

\section{Engineering modeling}

Based on the review presented in Section 1 for vibro-impact phenomena, Fig. 1 shows a flowchart for modeling and analysis of general physical systems. Using signal processing it is possible to record some features of the empirical behavior of some equipment, whose mathematical model is obtained with the aid of physical laws and assumptions, in the form of a differential equation in most cases. Differential equations can be solved either analytically or numerically. While approximated numerical solutions can always be obtained through discretization of the equation's independent variables, the existence of closed-form analytical solutions depends on the equation's mathematical properties. For nonlinear models, one can establish certain mathematical assumptions, and then obtain an approximated analytical solution under strict conditions. 
Experimental, numerical and analytical results are discussed in this work. In order to produce a concise discussion, focused on the vibro-impact behavior, the singledegree of freedom model shown in Fig. 2 will be used to illustrate the application of different analytical techniques to non-smooth systems. This is a nondimensional model, time-normalized using its linear natural frequency, and its equation of motion is given by:

$$
\ddot{q}+2 \beta \dot{q}+q=\Omega^{2} B \sin (\Omega t), \quad \text { if } \quad q<\Delta,
$$

where $\beta, \Omega, B$ and $\Delta$ are the damping ratio, forcing frequency, forcing amplitude and gap width, respectively. $q(t)=y(t)-b(t)$ is the relative displacement between the inertial coordinates $y(t)$ and $b(t)=B \sin (\Omega t)$. The impact condition is:

$$
q\left(t_{-}\right)=\Delta \Rightarrow\left\{\begin{array}{l}
q\left(t_{+}\right)=q\left(t_{-}\right), \\
\dot{q}\left(t_{+}\right)=-R \dot{q}\left(t_{-}\right),
\end{array}\right.
$$

where $R$ is the coefficient of restitution and $t_{+}$and $t_{-}$are the time instants after and before impact, respectively. From now on terms such as $q\left(t_{ \pm}\right)$will be represented by $q_{ \pm}$. The impact condition, Eq. (2), can be rewritten as:

$$
\dot{q}_{+}-\dot{q}_{-}=-(1+R) \dot{q}_{-}
$$

which shows that the impacts create a velocity jump which has at least the same order of magnitude as the pre-contact velocity for $R=0$ or twice that for elastic impacts, $R=1$.

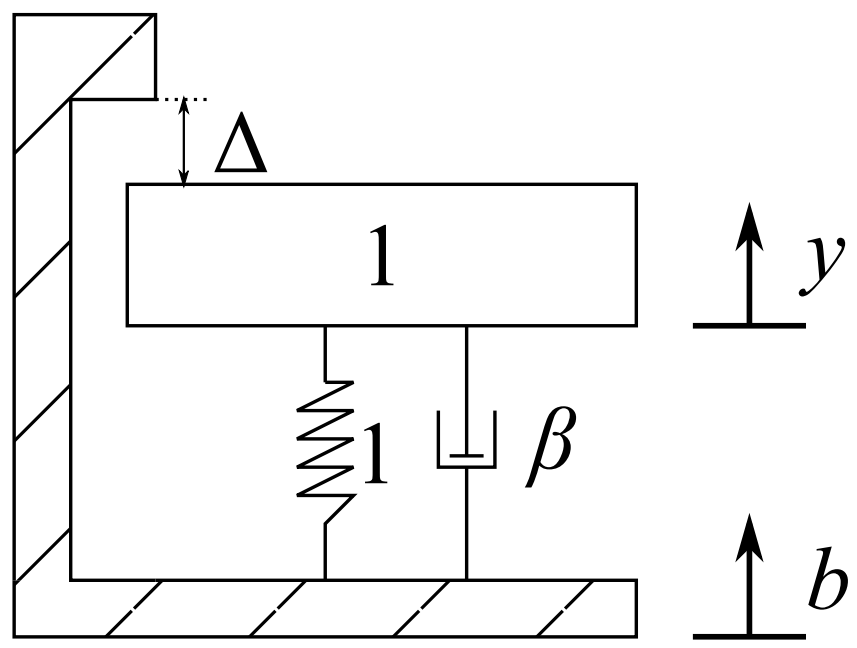

Figure 2: Base-excited SDOF system with unilateral constraint. $b(t)=B \sin (\Omega t)$

Model parameter values are $\beta=15 \times 10^{-3}$ and $B=0.5 \times 10^{-3}$, unless otherwise specified. The forcing frequency varies in the range $\Omega=1.5$ to 2.5 . Numeric values for gap width and the coefficient of restitution will be given later on. 


\section{Numerical Analysis}

Numerical simulation is the most common tool to obtain approximate solutions for arbitrary nonlinear models. While Equation (1) is continuous, the impact condition, given by Eq. (2), produces a discontinuity which prevents the direct use of classical numerical schemes, which should be integrated with auxiliary schemes to produce reasonable results. One of the most common approaches is to use an event-handling scheme to identify the occurrence of impacts, using the post-contact state as initial conditions to the ODE solver. Alternatively, one can use coordinate transformations to reduce or eliminate the velocity jump due to impacts. In this section, both event-handling techniques and coordinate transformations will be used separately or combined and their results will be compared. Results from analytical techniques will be added to the comparison in Section 4 .

Time-integration of the model equations was performed using the built-in MAT$\mathrm{LAB}^{\circledR}$ ODE solver ode 23 with relative and absolute error tolerances of RelTol = $1.0 e-4$ and Abstol $=1.0 e-8$. The option 'events' is used to handle the transition between impacting and non-impacting regimes when necessary.

\subsection{Direct Numerical Simulation}
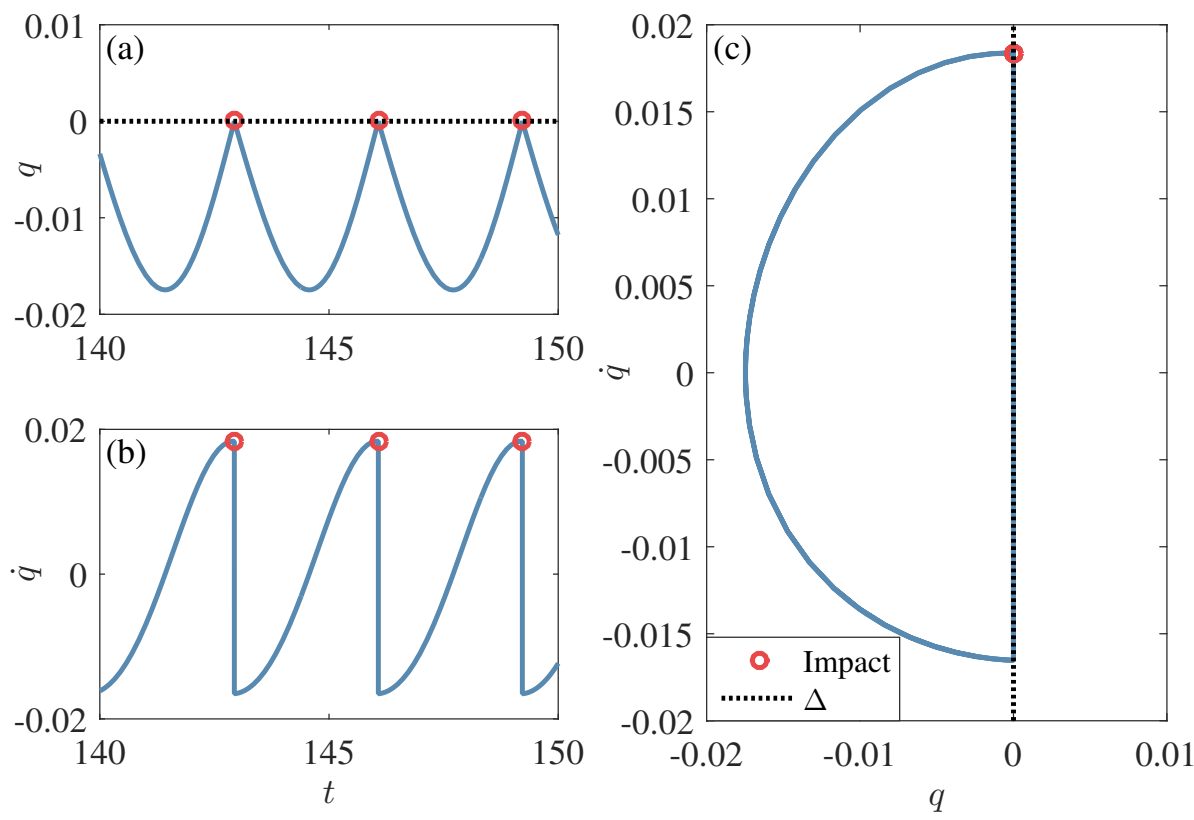

Figure 3: Results from direct numerical simulation: time series for (a) position and (b) velocity, and (c) phase-portrait.

The results from direct numerical time-integration of Eqs. (1) and (2) using the nominal parameters for $R=0.9$ and $\Delta=0$ are shown in Fig. 3 The stop location 
is represented by a straight dotted black line and impact states are marked with red circles.

Non-smooth model components such as Eq. (2) are the main barrier to overcome when dealing with discontinuous systems. There are many works devoted to bypassing this problem from both numerical [47,-49, 58] and analytical [23, 35, 59] perspectives. A combined version of both approaches is presented in [46], which applies nonsmooth coordinate transformations to a specific problem in order to weaken discontinuous terms and then performs numerical simulation on the transformed problem. This approach is briefly discussed in the next two sub-sections.

\subsection{Numerical Simulation using Zhuravlev's Transformation}

The following Zhuravlev's transformation [37], was developed specifically for unilateral vibro-impact problems. It consists of unfolding the displacement $q(t)$ limited by a unilateral constraint at $q=\Delta$ into an unconstrained variable $z(t)$, shifting the barrier to $z=0$ as shown in Fig. 4. Notice that $z$ is smooth and change its sign at the points where $q$ is non-smooth. Mathematically, the transformation is given by [37]:

$$
q(t)=\Delta-|z(t)|,
$$

where $z(t)$ is called unfolded variable. The first and second time-derivatives of Eq. (4) during non-impact times $(z \neq 0)$ are:

$$
\begin{aligned}
& \dot{q}=-\dot{z} \operatorname{sgn}(z), \\
& \ddot{q}=-\ddot{z} \operatorname{sgn}(z),
\end{aligned}
$$

where sgn is the sign function. Substituting Eq. (4) and its derivatives on Eq. (1), one obtains:

$$
\ddot{z}+2 \beta \dot{z}+z=\operatorname{sgn}(z)\left(\Delta-\Omega^{2} B \sin (\Omega t)\right), \quad \text { if } \quad z \neq 0 .
$$

Knowing that $z_{+} z_{-}<0$, the impact condition, given by Eq. (2), can be rewritten as:

$$
\begin{aligned}
\dot{z}_{+} & =R \dot{z}_{-}, \quad \text { if } \quad z=0, \\
\dot{z}_{+}-\dot{z}_{-} & =-(1-R) \dot{z}_{-} .
\end{aligned}
$$

The last expression shows that the impacts create a velocity jump on the unfolded variable proportional to $1-R$ which is small for near elastic impacts, $R \approx 1$. Although this jump gets bigger for $R \rightarrow 0$ it is always smaller than the one obtained from the original coordinates of the problem, given by Eq. (3).

One can avoid the use of the impact condition, Eq. (8) by inserting the velocity jump due to impacts directly into Eq. (7) using Dirac's delta function, which becomes [46]:

$$
\ddot{z}+2 \beta \dot{z}+z+(1-R) \dot{z}|\dot{z}| \delta_{-}(z)=\operatorname{sgn}(z)\left(\Delta-\Omega^{2} B \sin (\Omega t)\right),
$$

where, according to [46], $\delta_{-}(\cdot)$ is a modified version of Dirac delta function, whose singularity occurs on the left of zero, instead of at zero. In this case, the resulting model still contains a singularity, which is now multiplied by a small factor $1-R$, if 


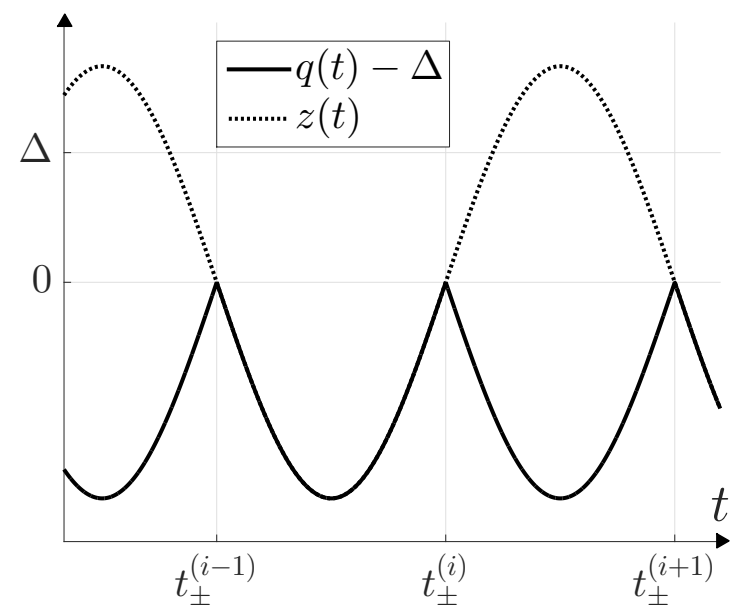

Figure 4: Displacement $q(t)$ and unfolding variable $z(t)$.

$R \approx 1$. This can be done using the model original coordinates as well, see [26, 41]. In this case, one has to interpret the model in terms of the distribution theory, due to the presence of a summation of classical Dirac delta functions $(1+R) \dot{q} \delta\left(t-t_{j}\right)$ instead of $(1-R) \dot{z}|\dot{z}| \delta_{-}(z)$, where $t_{j}$ represents the $j$-th impact instant.

From a numerical perspective, there are two ways to handle the term $\delta_{-}(z)$ in Eq. (10): replace it by a finite smooth approximation, or neglect it under the assumption that $R \approx 1$ and $1-R \approx 0$. The first alternative leads to additional model parameters to describe the smoothed function, while the second one eliminates the possibility of examining the influence of small variations of the coefficient of restitution on the model response. Alternatively, one can use Eq. (7) with an event handling algorithm for Eq. (8), whose smaller velocity jump (compared to Eq. (8)) should decrease computation efforts. After simulation, the model original coordinates can be obtained using Eqs. (4) and (5).

Despite the persistence of the impact rule on the transformed model, the velocity jump caused by the impacts has been decreased. That can be observed in Fig. 5(b) which shows the velocity and its transformed counterpart. A minor problem of this approach, which could not be solved by varying the ODE's solver properties, is that the original velocity presents an additional jump immediately after impact, see the bottom part of Fig. $5(\mathrm{~b}, \mathrm{c})$. 

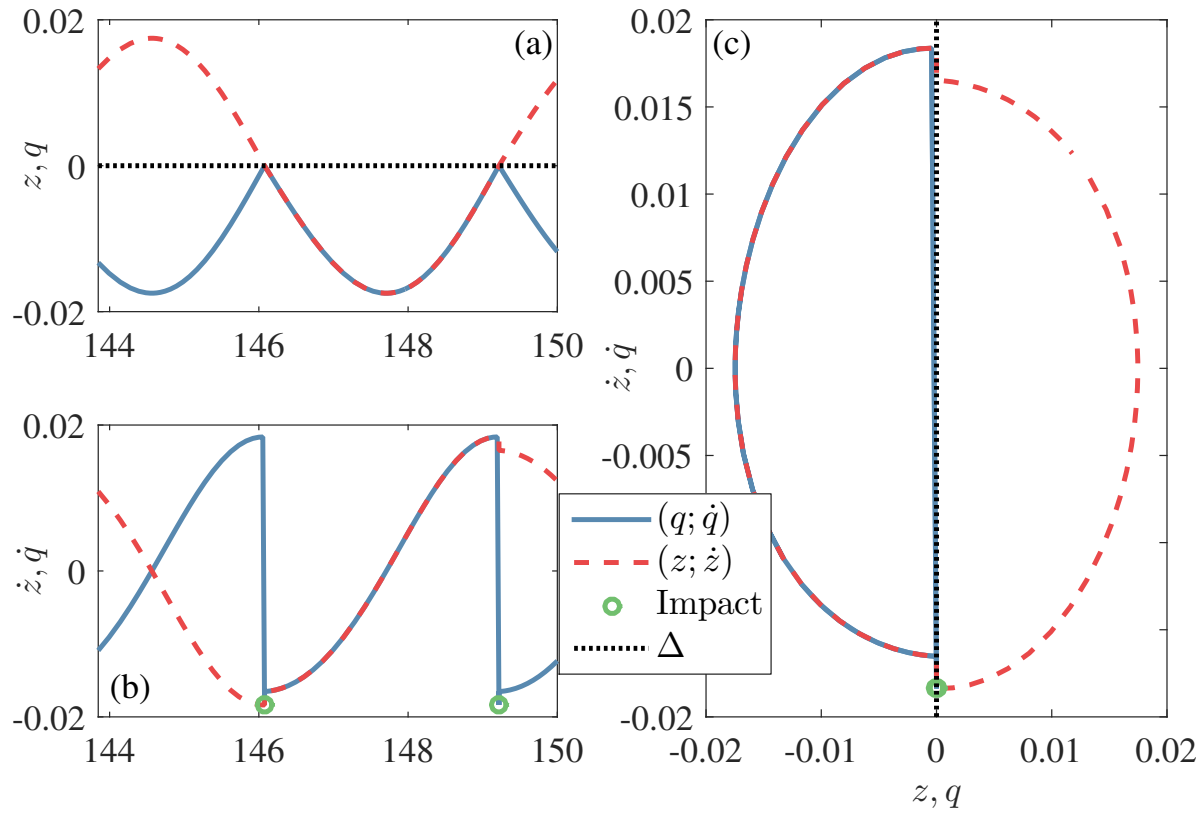

Figure 5: Numerical simulation using Zhuravlev transformation showing physical and transformed coordinates. Position and velocity in time( $a, b)$ and phase-portrait (c).

\subsection{Numerical Simulation using Ivanov's Transformation}

Ivanov's transformation can be seen as an extension of Zhuravlev's substitution, which is also restricted to models with unilateral constraint. Ivanov [38] proposed an additional transformation for the velocity term, which is not directly related to the displacement variable:

$$
\begin{aligned}
& q(t)=\Delta-|z(t)|, \\
& \dot{q}(t)=-K_{N} s(t) \operatorname{sgn}(z(t)), \\
& K_{N}=1-K \operatorname{sgn}(z(t) s(t)), \quad K=\frac{1-R}{1+R} .
\end{aligned}
$$

${ }_{227}$ A downside of this approach is the lack of physical interpretation for the new variable ${ }_{228} s(t)$. This change of coordinates reduces to Zhuravlev's if $R \approx 1$, in which case $s=\dot{z}$. 229 While Zhuravlev's transformation decreases the velocity jump during impacts, the term $K_{N}$ in Ivanov's coordinate conversion inserts the energy losses related to the collisions directly in the transformation, eliminating the need for an impact condition.

Writing Eq. (1) on state-space form and substituting Eqs. (11) and (12), leads to:

$$
\begin{aligned}
& \dot{z}=K_{N} s, \\
& \dot{s}=-2 \beta s+\frac{1}{K_{N}}\left(\left(\Delta-\Omega^{2} B \sin (\Omega t)\right) \operatorname{sgn}(z)-z\right) .
\end{aligned}
$$


One can obtain Eq. (7) from Eqs. (14) and (15), by realizing that $K_{N}$ is constant in each quadrant of the $(z, s)$-plane, $z, s \neq 0$. However, this removes the energy loss due to impacts from the model. Thus, Ivanov transformed systems are usually described as a set of first-order differential equations.

The steady-state response of Eqs. (14) and (15) using numerical simulation can be seen in Fig. 6 The problem's original coordinates are obtained after simulation using Eqs. (11) and (12). While $s$ is symmetric about the time-axis, $\dot{q}$ it is not, see Fig. 6. (c). The displacement variables $q$ and $z$ are coincident for $q \leq \Delta$. Due to the elimination of the impact condition, the impact states are not explicitly recorded during simulation.
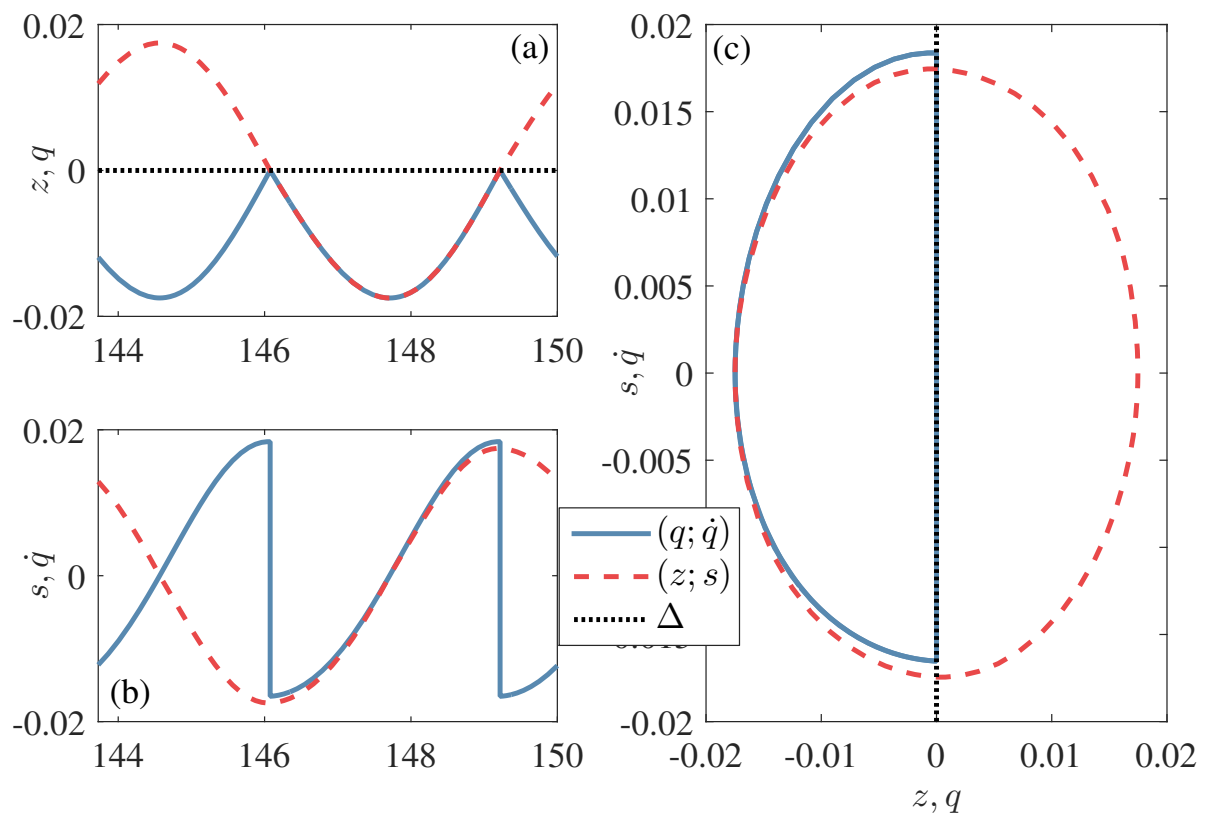

Figure 6: Numerical simulation using Ivanov transformation showing physical and transformed coordinates. Position and velocity in time $(a, b)$ and phase-portrait (c).

The results obtained from the three simulation approaches discussed in this section can be seen in Fig. 7. Observing the phase-portrait Fig. 7(a) one can see that the results are overlapped, except during contact, where Zhuravlev's response (red dashed line) has an additional velocity jump after impact and is not parallel to the $\Delta$-line. In the frequency domain Fig. 7(b), the three methods produce similar results, being overlapped. Using Ivanov and Zhuravlev transformations prior to numeric integration reduces the simulation time by 80 and $70 \%$ respectively. 

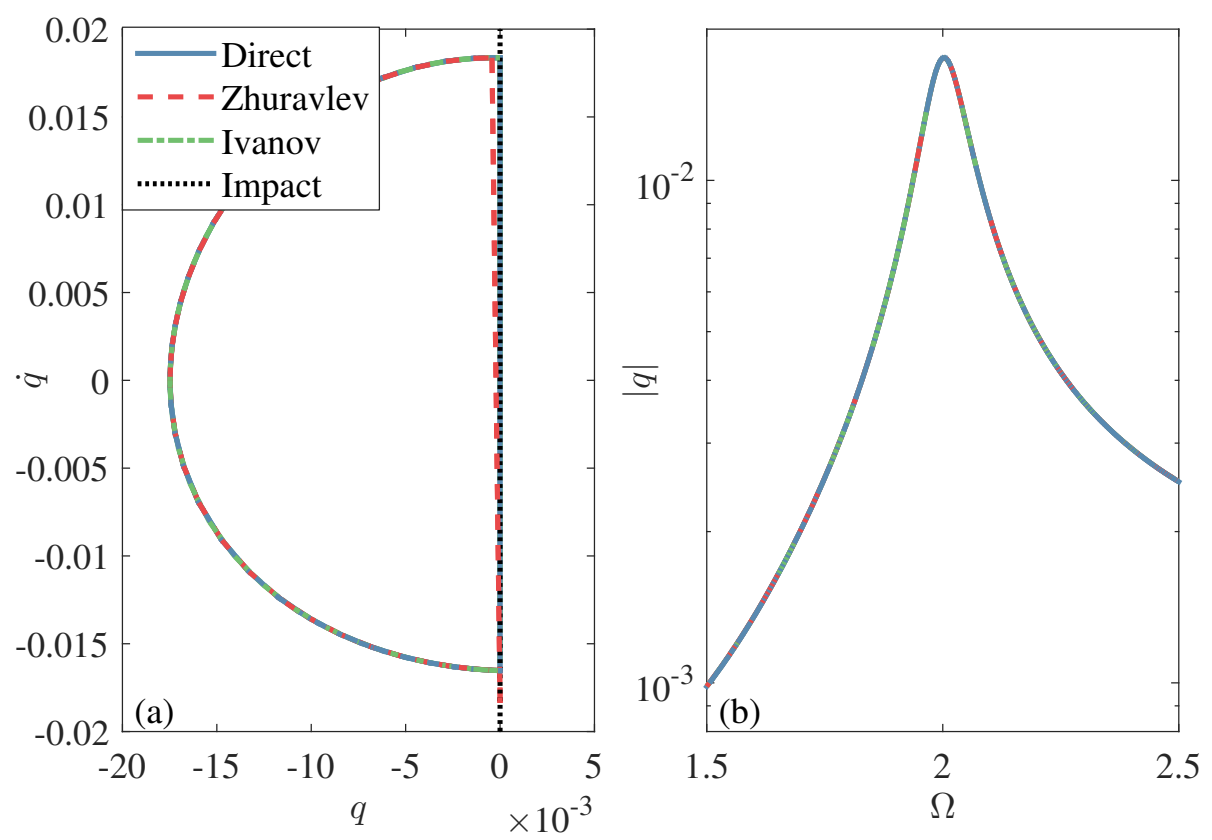

Figure 7: Results obtained using direct numerical simulation and together with Zhuravlev and Ivanov transformations. (a) Phase-portrait for $\Omega=2$ (b) and frequency response.

\section{Analytical Techniques}

\subsection{Averaging and Zhuravlev's transformation}

To obtain approximate steady-state periodic solutions for the vibro-impacting oscillator given by Eqs. (1) and (2) one can combine Zhuravlev's transformation for unilateral VI problems Eq. (4) with standard averaging. This section briefly describes this process, which is detailed discussed in [23, 37, 39].

The coordinate transformed system is the same as Eqs. (7) and (8) and is shown again here to facilitate reading:

$$
\begin{aligned}
\ddot{z}+2 \beta \dot{z}+z & =\operatorname{sgn}(z)\left(\Delta-B \Omega^{2} \sin (\Omega t)\right), & & \text { if } z \neq 0 \\
\dot{z}_{+}-\dot{z}_{-} & =-(1-R) \dot{z}_{-}, & & \text {if } z=0
\end{aligned}
$$

Admitting that the impacts are almost elastic $(R \approx 1)$ and that the damping ratio $\beta$, gap width $\Delta$ and forcing amplitude $B$ are small, one can apply the Van der Pol transformation $(z=Q \sin \varphi(t)$ and $\dot{z}=Q \cos \varphi(t), Q=Q(t)>0)$ in Eqs. (16) and (17). After some mathematical manipulations, one can show that single-sided vibro-impact systems such as Eqs. (16) and (17) are under resonance when excited at even multiples of their fundamental natural frequency, $\Omega \approx 2 k$, for $k=1,2,3, \ldots$ Also, one can obtain the following quadratic polynomial in $Q$, representing the frequency-amplitude relationship, [39]:

$$
P(Q)=g_{2} Q^{2}+g_{1} Q+g_{0}=0
$$


where:

$$
\begin{array}{r}
g_{2}=\pi^{2}\left(\sigma_{k}^{2}+\beta_{k}^{2}\right), \quad g_{1}=4 \pi \Delta \sigma_{k}, \\
g_{0}=4\left(\Delta^{2}-\left(B_{k} \Omega^{2}\right)^{2}\right), \quad B_{k}=\frac{B}{4 k^{2}-1} \\
\sigma_{k}=\frac{\Omega}{2 k}-1, \quad \beta_{k}=\beta_{\mathrm{eff}} / 2 k, \quad \beta_{\mathrm{eff}}=\beta+\frac{1-R}{\pi} .
\end{array}
$$

The detuning parameter $\sigma_{k}$ indicates how close the forcing frequency $\Omega$ is to the resonances $2 k$. The term $\beta_{\text {eff }}$ represents the system's effective damping, which is a combination of $\beta$ and $R$. The phase is given by:

$$
\begin{aligned}
\varphi(t) & =\frac{\Omega t}{2 k}+\theta, \\
\theta & =\arctan \left(\frac{\pi \sigma_{k} Q+2 \Delta}{-\pi \beta_{k} Q}\right) \frac{1}{2 k} .
\end{aligned}
$$

As $g_{2}$ is positive, $P(Q)$ has a unique global minimum point at:

$$
Q^{*}=-\frac{g_{1}}{2 g_{2}}=\frac{-2 \sigma_{k} \Delta}{\pi^{2}\left(\sigma_{k}^{2}+\beta_{k}^{2}\right)} .
$$

As the amplitude $Q$ is positive by definition, Eq. (24) implies that $\sigma_{k} \Delta<0$. So, as pointed out in [37], if the system is tightened $(\Delta<0)$, stable solutions should be seen after crossing resonance, where $\sigma_{k}>0$. Similarly, if the gap is positive, one should expect stable solutions before crossing resonances. One can show that $Q^{*}$ is also a lower stability boundary for the amplitude variable through eigenvalue analysis of the amplitude-phase $(Q, \theta)$ system of ODE's generated during averaging analysis [39].

A stability boundary can be defined in terms of the gap width $\Delta$ as well. Analyzing the discriminant of Eq. [18, which is given by:

$$
D[P(Q)]=g_{1}^{2}-4 g_{0} g_{2}=h_{2} \Delta^{2}+h_{0},
$$

with:

$$
h_{2}=-\left(4 \pi \beta_{k}\right)^{2}, \quad h_{0}=\left(4 \pi B_{k} \Omega^{2}\right)^{2}\left(\sigma_{k}^{2}+\beta_{k}^{2}\right)
$$

279 To obtain real-valued amplitude solutions, $D[P(Q)]$ should be positive, therefore the 280 gap's absolute value $|\Delta|$ cannot exceed the following boundary value:

$$
\Delta_{M A X}=\sqrt{\frac{h_{0}}{-h_{2}}}=B_{k} \Omega^{2} \sqrt{\left(\frac{\sigma_{k}}{\beta_{k}}\right)^{2}+1} .
$$

As $B_{k}$ is positive, $\Delta_{M A X}$ is also positive, having a point of minimum at resonance $\Omega=2 k$. The advantage of defining the stability boundary in terms of the gap instead of amplitude is that while $Q$ is a value to be found, $\Delta$ is a design parameter in some applications. Finally, one could obtain a stability boundary using the forcing frequency 
$\Omega$ and Eq. 25], but the resulting $\Omega$-polynomial will be of fourth degree, implying more complex expressions.

The equivalence between stability boundaries for amplitude and gap width is shown in Fig. 8(b,c) for $\Delta=0.7 \times 10^{-3}$. In Fig. 8 (a), forward and backward numeric sweeps are able to follow only the upper branch of the frequency-response curve obtained using averaging, indicating the stability of this branch and agreeing with the stability boundary. Based on Eq. (4), the amplitude $Q$ is obtained by subtracting the minimum steady state value from $\Delta$, i.e., $Q=\Delta-\min \left(q_{s s}\right)$, where $q_{s s}$ is the steady-state response.
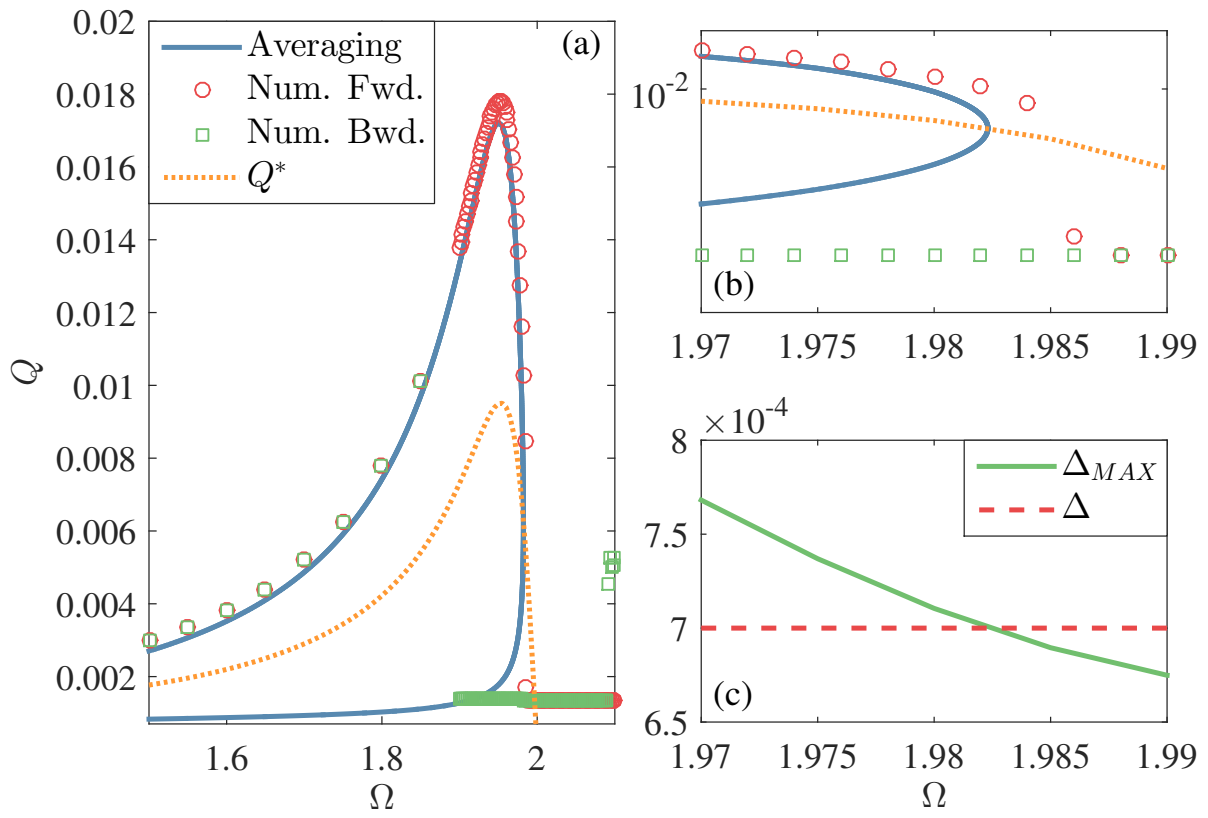

Figure 8: (a) Frequency response curve and minimum amplitude for $\beta=15 \times 10^{-3}$ and $\Delta=0.7 \times 10^{-3}$, (b) zoom on (a) and (c) gap boundary value.

Despite being not possible to obtain expressions like Eqs. (24) and (27) for more complex systems, it is still possible to use the parameter relations to get qualitative insight about the stability boundaries. For instance, the presence of $\beta_{k}$ on the denominator of Eqs. (24) and (27), indicates that decreasing the effective damping increases both stability thresholds. At first, this sound counter-intuitive since adding damping usually improves a system's stability characteristics, but as lower damping increases the response amplitude, it seems reasonable that its stability boundary expands as well. The influence of damping on frequency response can be seen by comparing Figs. 8 and 9 for $\beta=15 \times 10^{-3}$ and $1 \times 10^{-3}$ respectively. As expected both amplitude and its stability boundaries increase for smaller damping.

The influence of $\Delta$ and $R$ on the frequency response is shown in Fig. 10. In Fig. 10.a), one can see that the location of the peak of amplitude response moves from after $\Omega=2$ to before this value as the gap width $\Delta$ passes from tight (negative) to loose (positive gap) configuration. The maximum amplitude is also proportional to the coefficient of 


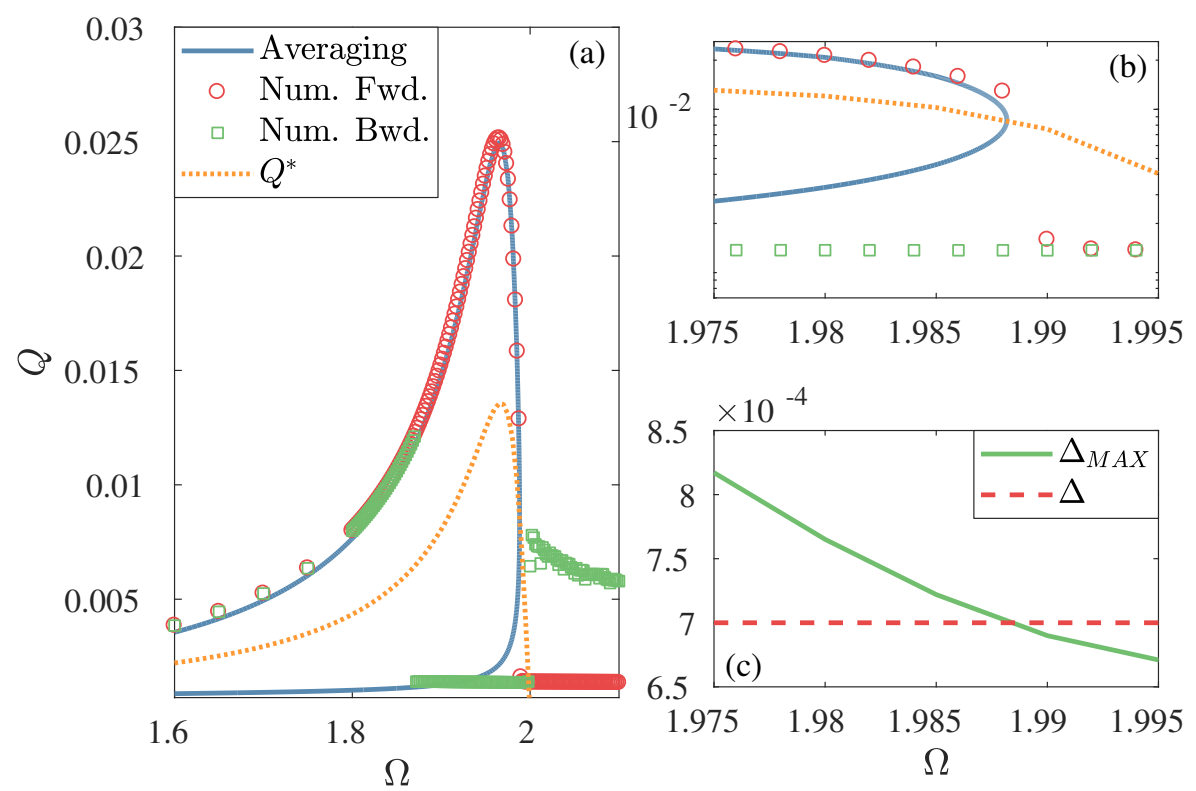

Figure 9: (a) Frequency response curve and minimum amplitude for $\beta=1 \times 10^{-3}$ and $\Delta=0.7 \times 10^{-3}$, (b) zoom of (a), and (c) gap boundary value.

restitution, Fig. 10(b), due to the decrease in energy dissipation due to impacts for higher $R$.

One of the main conditions for using the results discussed in this section is that the impacts are almost elastic $(R \approx 1)$. The extent of this condition is analyzed in Fig. 11 for different values of $R$, where one can see that the agreement between averaging and numerical simulations decrease with the coefficient of restitution, having a $16 \%$ difference in amplitude for $R=0.6$ at $\Omega=2$. 


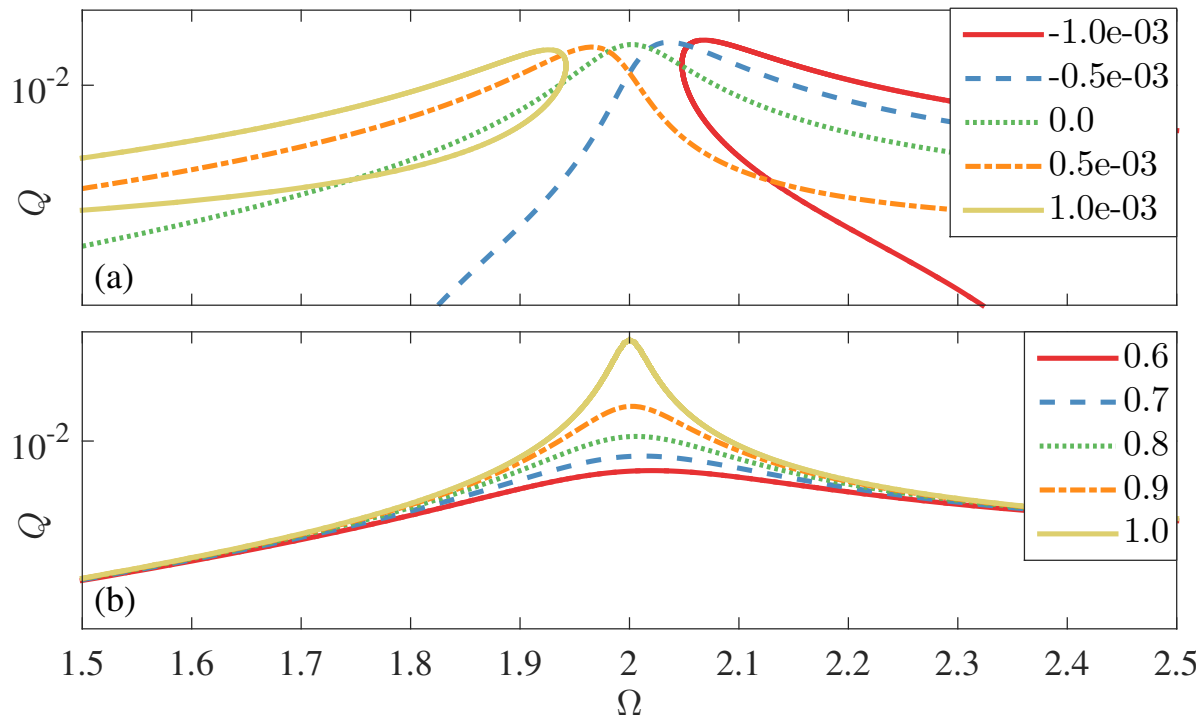

Figure 10: Influence of (a) gap width $\Delta$ and (b) coefficient of restitution $R$ on the frequency response using averaging.
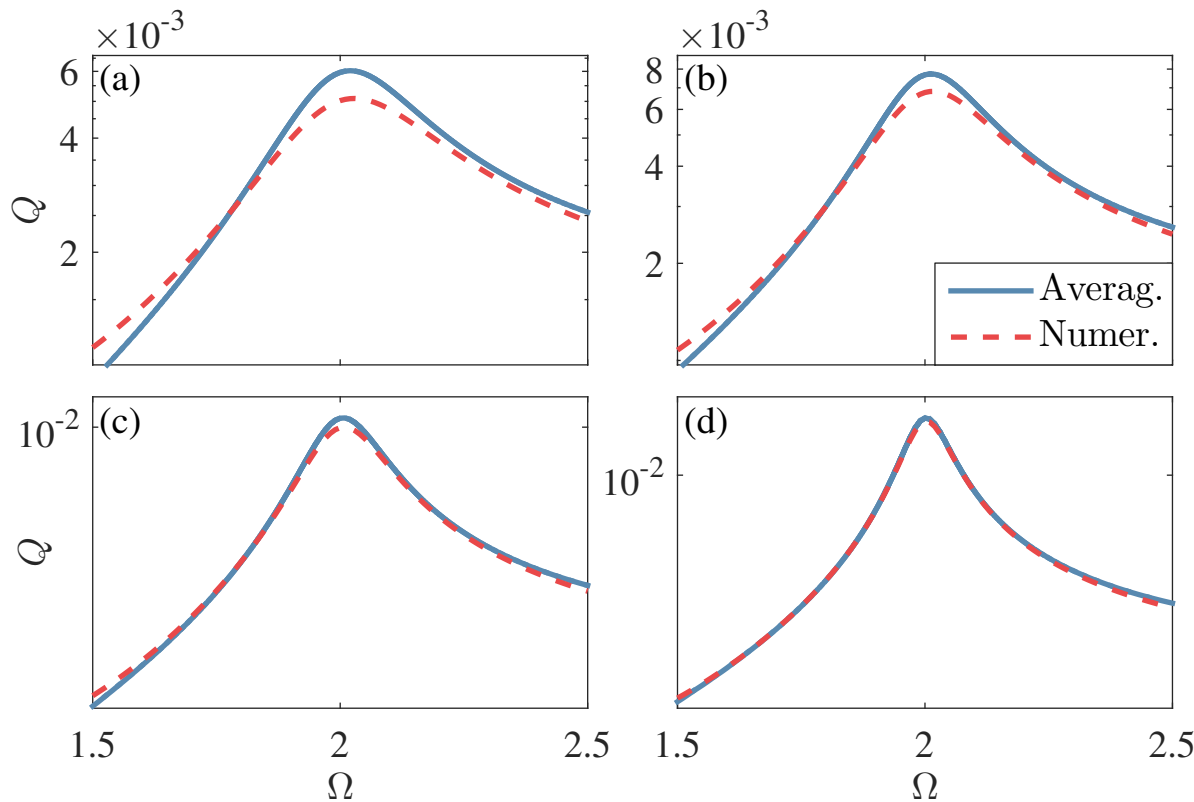

Figure 11: Comparison of numeric and averaging frequency responses for (a) $R=0.6$, (b) $R=0.7$, (c) $R=0.8$ and (d) $R=0.9$. 


\subsection{Pointwise mapping}

Another way to obtain solutions for the vibro-impact model Eqs. (1) and (2), is to use the impact condition Eq. (2) to link the linear solutions of non-impacting regimes, given by:

$$
q(t)=e^{-\beta_{s} t}\left(A_{1}\left(X_{0}\right) \cos \left(\omega_{D} t\right)+A_{2}\left(X_{0}\right) \sin \left(\omega_{D} t\right)\right)+C \sin (\Omega t+\theta), \quad \text { if } \quad q<\Delta,
$$

where $X_{0}=\left(t_{0}, q_{0}, \dot{q}_{0}\right)$ and $\omega_{D}=\sqrt{1-\beta_{S}^{2}}$ represent the initial conditions and damped natural frequency for non-impacting configuration respectively.

The post-impact state from a previous collision $\left(t_{+}^{(i-1)}, \dot{q}_{+}^{(i-1)}\right)$ can be used as an initial condition for Eq. 28. Then, the next collision instant $t_{-}^{(i)}$ can be obtained by applying a root-finding algorithm to $q\left(t_{-}^{(i)}\right)=\Delta$. From that, the collision velocity $\dot{q}_{-}^{(i)}$ can be found by substituting $t_{-}^{(i)}$ on the derivative of Eq. 28 . The next post-impact velocity $\dot{q}_{+}^{(i)}$ can be found by using the impact relation Eq. (2) restarting the cycle, as illustrated in Fig. 12 .
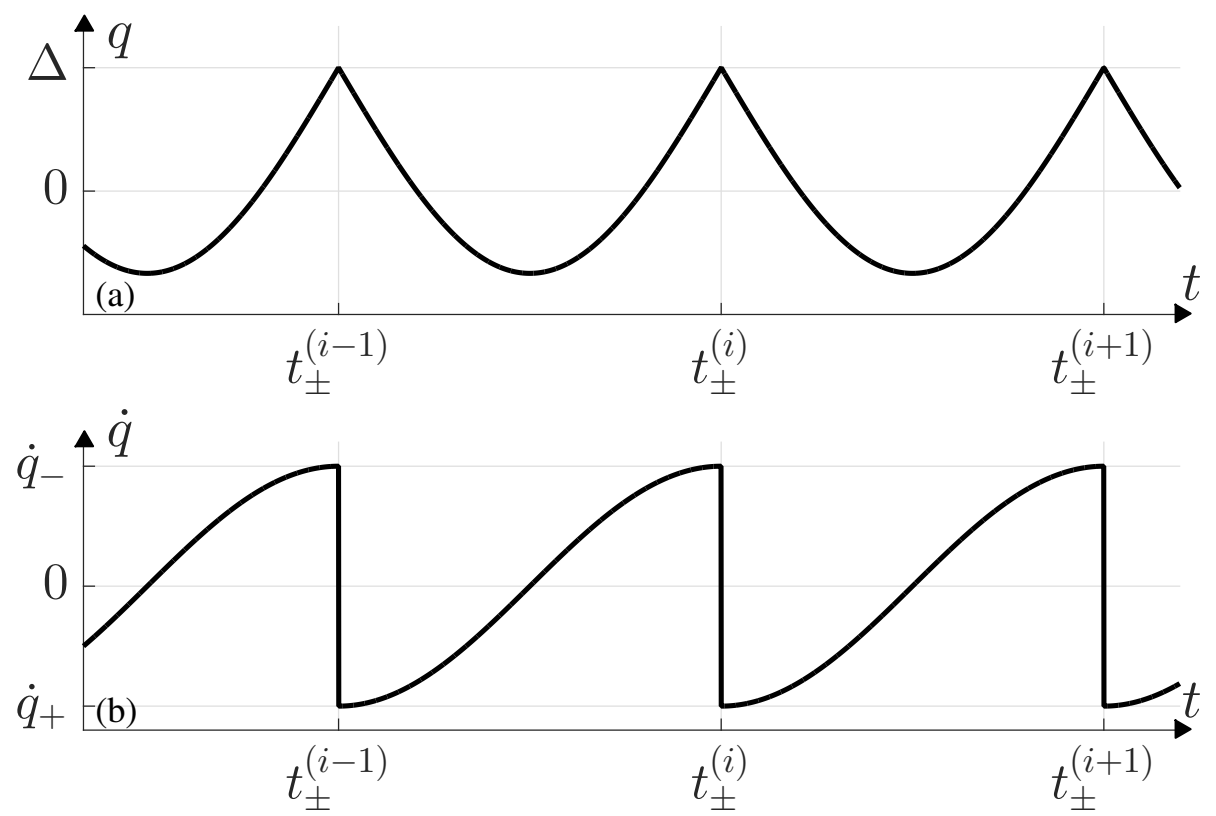

Figure 12: (a) displacement and (b) velocity time-series showing pre- and post-impact instants and velocities, $\left(t_{ \pm}^{(j)}, \dot{q}_{ \pm}^{(j)}\right)$.

326 Some drawbacks of this approach are that it:

327 - does not provide any expression for the system's resonances;

${ }_{328}$ - is not purely analytical, requiring a root-finding algorithm to work;

329 - cannot be used if other nonlinearities are also present; 
An exception to the last item is given by Eq. (7), which is linear for $z$ strictly positive/negative, enabling the application of pointwise mapping to Eqs. (7) and (8). In this case, the solution would be similar to Eq. (28) with an additional term to account for the particular solution to $\Delta \operatorname{sgn}(z)$.

The procedure described above was used by Shaw and Holmes [1] to derive a twodimensional discrete map of the pre-contact instant and velocity, $\left(t_{-}^{(i+1)}, \dot{q}_{-}^{(i+1)}\right)$. Given the need for root-finding techniques, it is not possible to obtain an analytical formula for this map. However, it is possible to analyze the stability of its equilibrium states using the map's Jacobian, which can be obtained analytically by deriving the linear solutions of the non-impacting configuration with respect to its initial conditions.

Considering that $t_{+}^{(i)} \approx t_{-}^{(i)}$ and $\dot{q}_{+}^{(i)}=-R \dot{q}_{-}^{(i)}$, the Jacobian's determinant and trace are given by:

$$
\begin{aligned}
\operatorname{det}(\boldsymbol{J}) & =-R \frac{\dot{q}_{+}^{(i-1)}}{\dot{q}_{-}^{(i)}} \exp \left(-2 \beta_{S} \tau\right), \\
\operatorname{tr}(\boldsymbol{J}) & =-\frac{\exp \left(-\beta_{S} \tau\right)}{\omega_{D} \dot{q}_{-}^{(i+1)}}\left(\left(N+R \beta_{S} \dot{q}_{-}^{(i)}\right) \sin \left(\omega_{D} \tau\right)+\right. \\
& \left.+\omega_{D}\left(\dot{q}_{+}^{(i-1)}-R \dot{q}_{-}^{(i)}\right) \cos \left(\omega_{D} \tau\right)\right), \\
\tau & =t_{-}^{(i)}-t_{-}^{(i-1)}, \\
N & =\gamma \tilde{V} \Omega^{2} \sin \left(\Omega t_{+}^{(i-1)}\right)-\beta_{S} \dot{q}_{+}^{(i-1)}-\Delta,
\end{aligned}
$$

where $\tau$ represents the non-contacting period. To analyze the stability of period- $n$ oscillations, Shaw and Holmes [1] assumed that:

$$
\begin{array}{r}
t_{-}^{(i)}=t_{-}^{(i-1)}+n T, \quad \dot{q}_{+}^{(i-1)}=-R \dot{q}_{-}^{(i-1)}, \\
\dot{q}_{-}^{(i)}=\dot{q}_{-}^{(i-1)}, \quad T=\frac{2 \pi}{\Omega},
\end{array}
$$

where $T$ is the period. With those assumptions Eqs. 29] and 30 become:

$$
\begin{aligned}
\operatorname{det}(\boldsymbol{J}) & =R^{2} \exp \left(-\beta_{S} n T\right), \\
\operatorname{tr}(\boldsymbol{J}) & =\frac{\exp \left(-\beta_{S} n T\right)}{\omega_{D} \dot{q}_{-}^{(i)}}\left(\left(R \beta_{S} \dot{q}_{-}^{(i)}+N\right) \sin \left(\omega_{D} n T\right)-2 \omega_{D} R \dot{q}_{-}^{(i)} \cos \left(\omega_{D} n T\right)\right), \\
N & =\gamma \tilde{V} \Omega^{2} \sin \left(\Omega t_{-}^{(i)}\right)+R \beta_{S} \dot{q}_{-}^{(i)}-\Delta,
\end{aligned}
$$

The frequency response for $\Delta=0.5 \times 10^{-3}$ obtained using numerical simulation and pointwise mapping is shown in Fig. 13(a). Its boxed region is shown in detail in Fig. 13(b), together with marks to indicate instability of period-1 oscillations. The stability was accessed using both full and simplified versions of the Jacobian's determinant and trace, given by Eqs. (29) and (30) and Eqs. (35) and (36), respectively. In this figure, one can see the full version of the Jacobian 's determinant and trace is able to identify more unstable orbits than its simplified version. Also, only two orbits were considered unstable by both methods and the oscillations at some frequencies were considered to be period-1 stable but presented non-periodic behavior, see the phase-portrait 

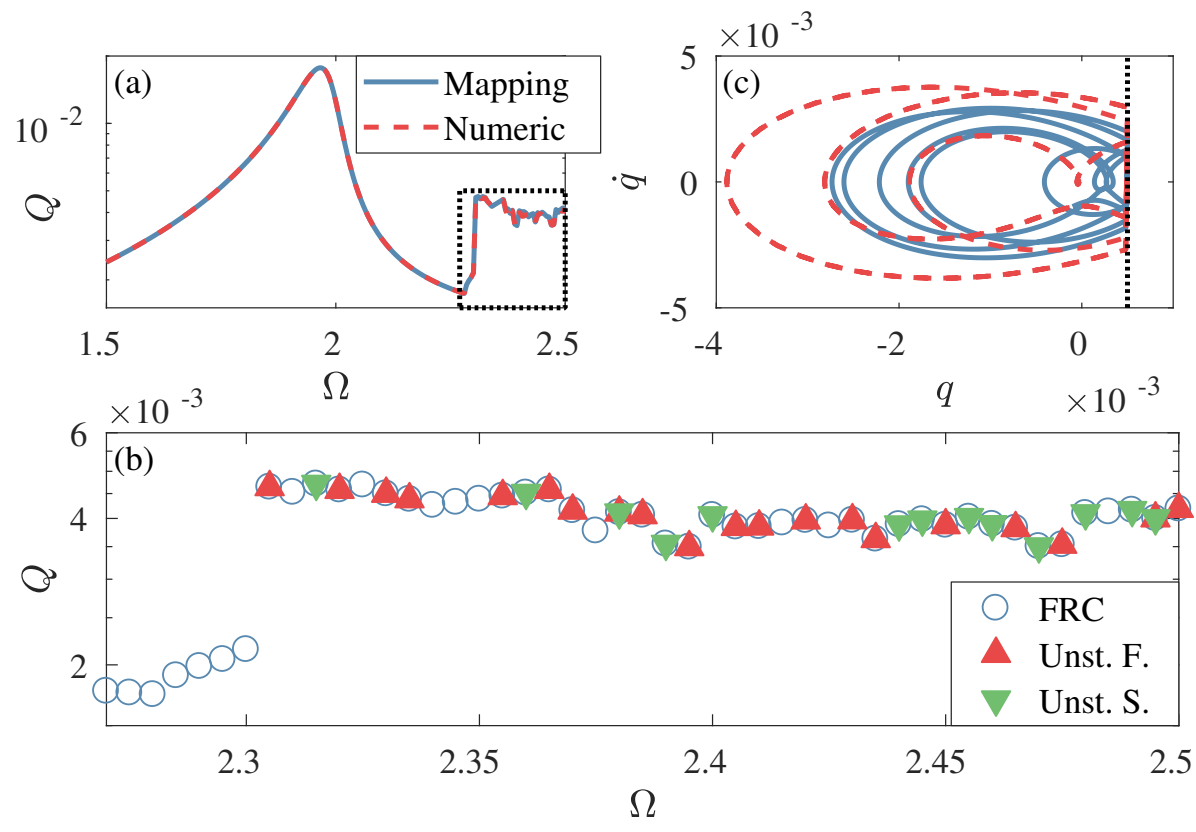

for $\Omega=2.35$ on Fig. 13 (c). Despite that, both approaches identify the beginning of instability at virtually the same frequency. The averaging analysis made in Section 4.1 is valid only in a small neighborhood of $\Omega=2$, being not able to identify the unstable region, which started for $\Omega \approx 2.3$. On the other hand, the stability equations provided by averaging analysis are more compact than those provided by pointwise mapping.

Figure 13: (a) FRC for $\Delta=0.5 \times 10^{-3}$. (b) Zoom at the boxed region shown in (a) indicating how the instability was accessed. (c) Phase-portrait at $\Omega=2.35$.

Observing Fig. 13,a) one can see that pointwise mapping gives the same quantitative results as numerical simulations for period-1 orbits. Both approaches also produce equivalent qualitative results for more complex behavior, as shown in Fig. 13. (c).

This approach can be used to piecewise linear systems as well. In this case, the transition between $t_{-}^{(i)}$ and $t_{+}^{(i)}$ is made by using the state at $t_{-}^{(i)}$ as an initial condition to the other linear system in contact configuration $(q \geq \Delta)$. The solution for $q\left(t_{+}^{(i)}\right)=\Delta$ provides the final contact instant $t_{+}^{(i)}$, which should be substituted on the time derivative of the contact configuration linear solution to find $\dot{q}_{+}^{(i)}$. Finally, the state at $t_{+}^{(i)}$ is used as initial condition for the non-contacting configuration linear solution, restarting the cycle. In this case, the system's Jacobian will be a product of the derivatives of contacting and non-contacting configurations with respect to their initial conditions.

A comparison between the results from averaging and pointwise mapping with numerical simulation is shown in Fig. 14 The differences between averaging (blue dashdotted line) and the other approaches can be easily noticed on phase-portrait, while the results are equivalent in the frequency domain, even far away from $\Omega=2$, where averaging should perform better. Regarding simulation time, the pointwise mapping is only $14 \%$ faster than direct numerical simulation, being slower to simulate than 
the non-smooth transformed models. That can be explained by its dependence on a root-finding procedure, whose results are more difficult to obtain in the transition between impacting and non-impacting behaviors. Averaging is $93 \%$ faster than direct numerical simulation because it does not need an iterative procedure.
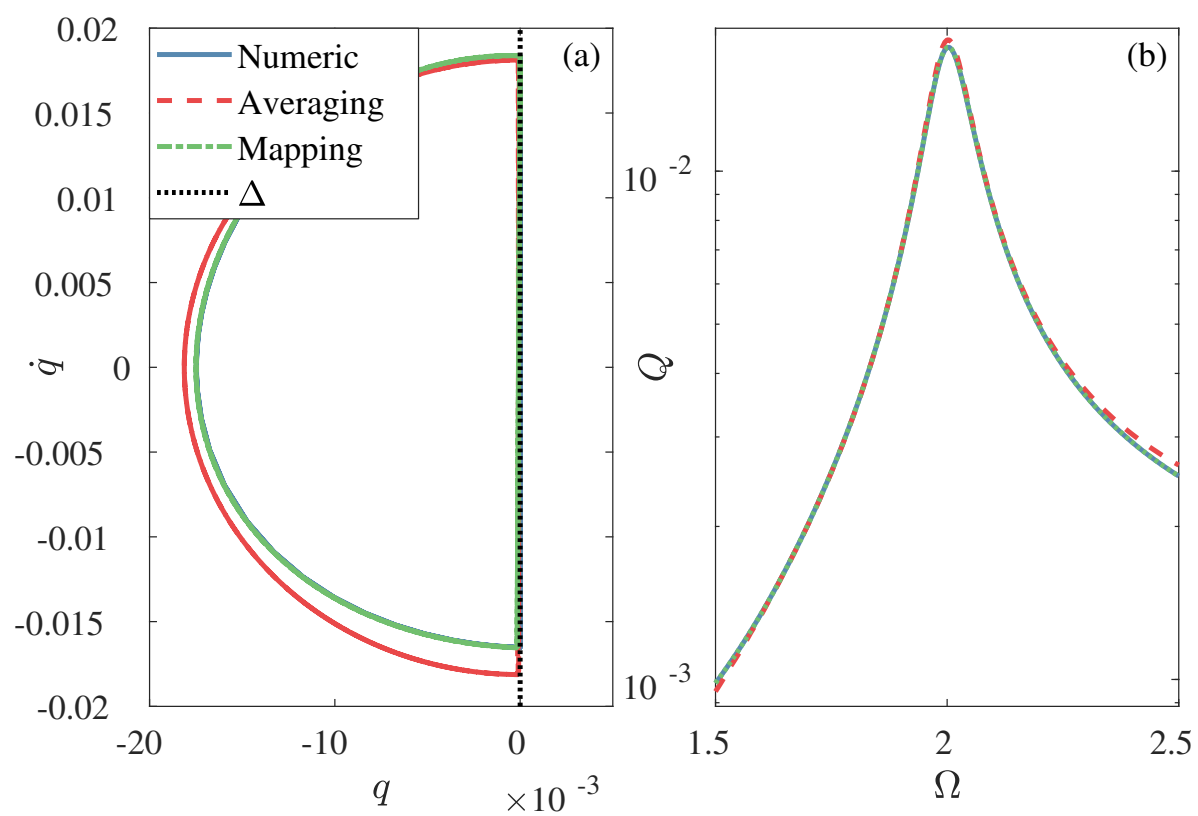

Figure 14: Use of different approaches to obtain (a) phase-portrait at $\Omega=2$ and (b) frequency response.

\section{Experimental setup}

The experimental setup has been described previously in [60 62] and is shown in Fig. 15 In Fig. 15.a, b) an electrodynamic shaker (1) [B\&K $\left.{ }^{\circledR} 4808\right]$ is used to apply a harmonic excitation to a platform (2), containing a cantilever beam with attached mass and a stop (5) located at the left of the mass, to restrain its lateral movement. The shaker is driven by a power amplifier $\left[\mathrm{B} \& \mathrm{~K}^{\circledR} 2712\right]$ and is connected to the platform by a stinger. The displacement of both platform and attached mass are measured by inductive sensors (3) [Pulsotronic ${ }^{\circledR}$ KJ4-M12MN50-ANU] and filtered by a Brickwall ${ }^{\circledR}$ filter [Wavetek 752A] hardware with low pass cut-off frequency of $500 \mathrm{~Hz}$. Data acquisition is performed at $5 \mathrm{kHz}$ sampling frequency using a dSPACE ${ }^{\circledR}$ DS1104 R\&D controller board.

The impacting beam can be seen in detail in Fig. 15. (c,d), where $U$ represents the beam's transversal displacement, $L_{S}=100 \mathrm{~mm}$ is the displacement's sensor axial location, $L_{m}=132 \mathrm{~mm}$ is the mass axial location, $L=180 \mathrm{~mm}$ is the beam's length, $\Delta_{C}$ is the gap width and $b(\tilde{t})$ is the base excitation. The attached mass $m$ weights $0.2 \mathrm{~kg}$ and it consists of 4 steel discs with $7 \mathrm{~mm}$ thickness and $35 \mathrm{~mm}$ diameter hold together with bolts. 

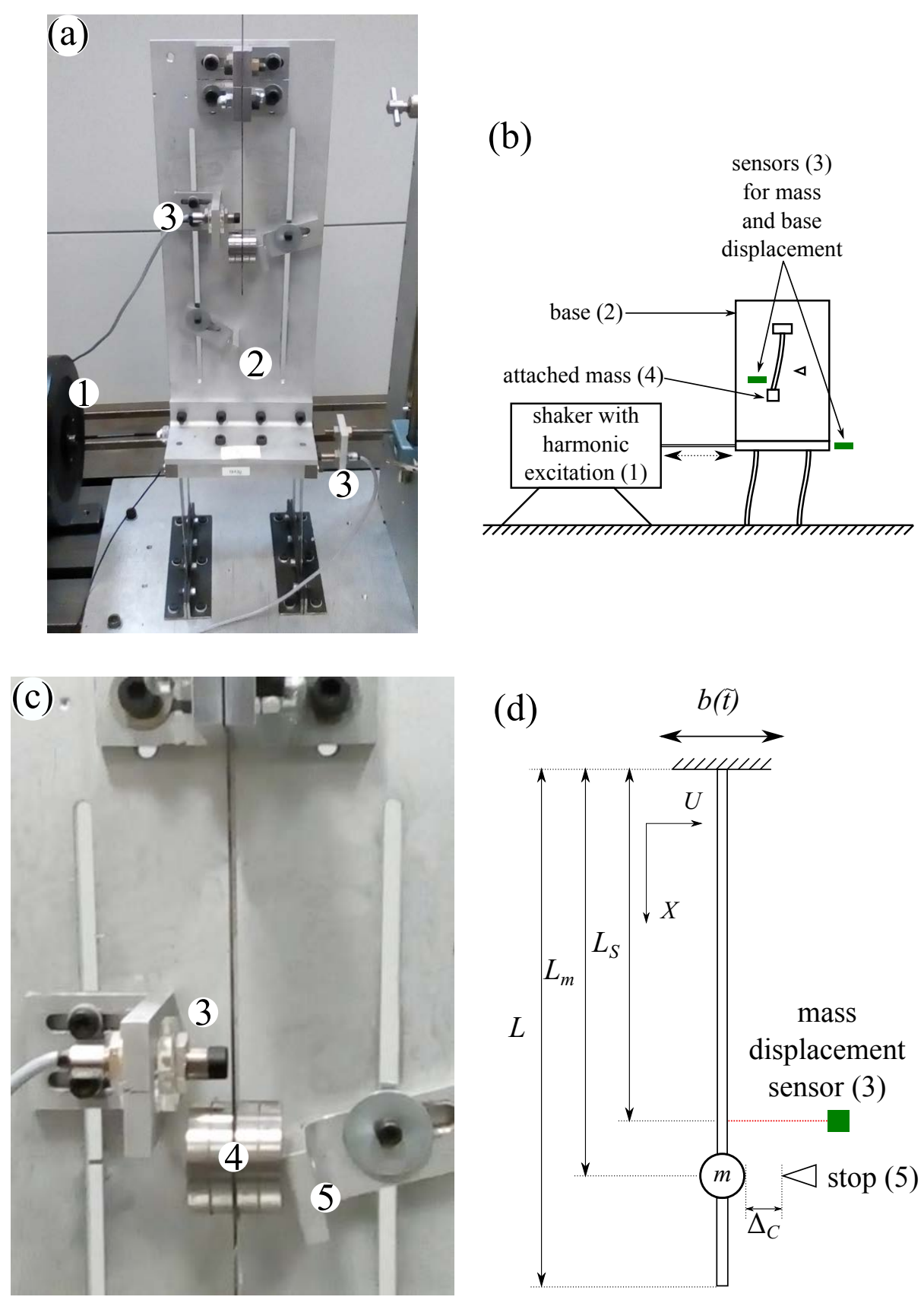

Figure 15: Experimental setup (a,c) and its schematic representation (b,d).

To avoid saturating the displacement sensors, measurement and impact locations 
$L_{S}$ and $L_{m}$ are not coincident. Thus the measured grazing width $\Delta_{G}$ will differ from the gap width $\Delta_{C}$ due to beam deflection, see Fig. 16. The measured grazing amplitude will be used to define the model's impact rule later on.

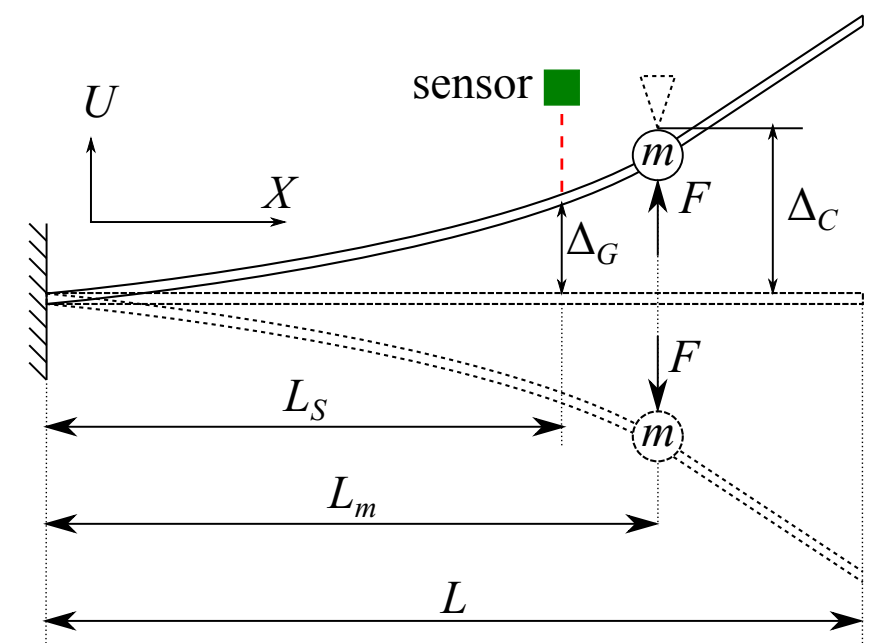

Figure 16: Deflection of a cantilever beam.

The setup's main natural frequencies are identified using experimental modal analysis with an accelerometer [B\& $\left.\mathrm{K}^{\circledR} 4397\right]$ and an impact hammer [B\& $\mathrm{K}^{\circledR}$ 8206]. Excitations were applied at $10 \mathrm{~mm}, 30 \mathrm{~mm}, 50 \mathrm{~mm}, 70 \mathrm{~mm}, 90 \mathrm{~mm}, 132 \mathrm{~mm}$ and $160 \mathrm{~mm}$ from the beam's clamped end and measured at $90 \mathrm{~mm}$ from the beam's clamped end.

Using a sampling frequency of $512 \mathrm{~Hz}$, the measured data is post-processed using $B \& K^{\circledR}$ PULSE frequency analyzer. The system's frequency response for an excitation at $50 \mathrm{~mm}$ from the beam's clamped end can be seen in Fig. 17, where one can identify four natural frequencies: $3.25 \mathrm{~Hz}, 8 \mathrm{~Hz}, 67 \mathrm{~Hz}$ and $98.75 \mathrm{~Hz}$ obtained with a resolution of $0.25 \mathrm{~Hz}$. At $3.25 \mathrm{~Hz}$ only the platform oscillates. The beam's first and second bending resonances occur at $8 \mathrm{~Hz}$ and $98.75 \mathrm{~Hz}$ respectively, with a torsional resonance at $67 \mathrm{~Hz}$.

From literature [1, 39, 63], it is known that SDOF single-sided vibro-impact systems present resonant behavior around even multiples of their natural frequency. Based on that the frequency range of interest in this work is lies between $14 \mathrm{~Hz}$ to $17 \mathrm{~Hz}$. Different gap configurations are also analyzed: pre-stressed $-0.1 \mathrm{~mm}$, loose $0.1 \mathrm{~mm}$, and neutral $0 \mathrm{~mm}$.

A compact view of this analysis is shown in Fig. 18 for different gap configurations: pre-stressed with $\Delta_{G} \approx-0.1 \mathrm{~mm}$ in Fig. 18,a); neutral with $\Delta_{G} \approx 0 \mathrm{~mm}$ in Fig. 18,b) and loose with $\Delta_{G} \approx 0.1 \mathrm{~mm}$ in Fig. 18 (c). There one can see an alternation between period one and two oscillations with the blank regions presenting more complex behavior, such as quasi-periodicity and chaos. The concentration of period-1 orbits before and after twice the system's fundamental resonance $\left(2 f_{n} \approx 15.5 \mathrm{~Hz}\right)$ for loose and prestressed configurations respectively is in accordance with the predictions made by [37] using Eq. (24) in Section 4.1. The pre-stressed configuration in Fig. 18(a), demands 

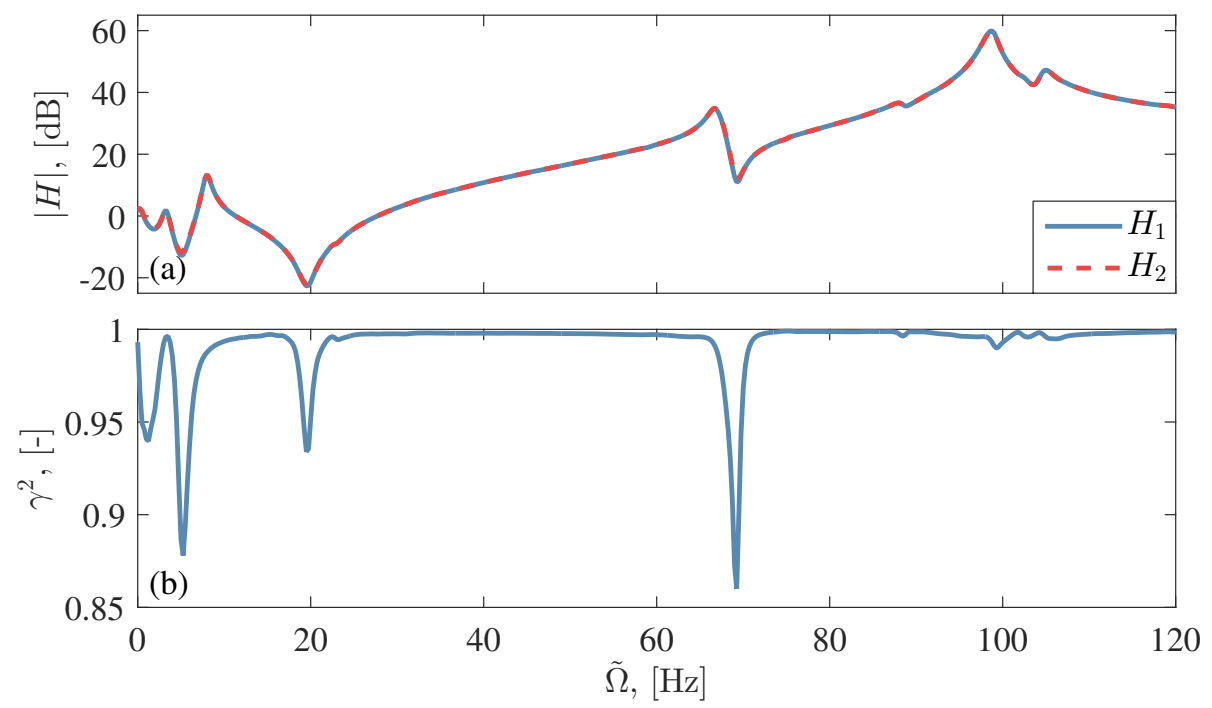

Figure 17: Frequency response: (a) amplitude, (b) coherence. oscillations are mainly present between $15 \mathrm{~Hz}$ to $15.5 \mathrm{~Hz}$.

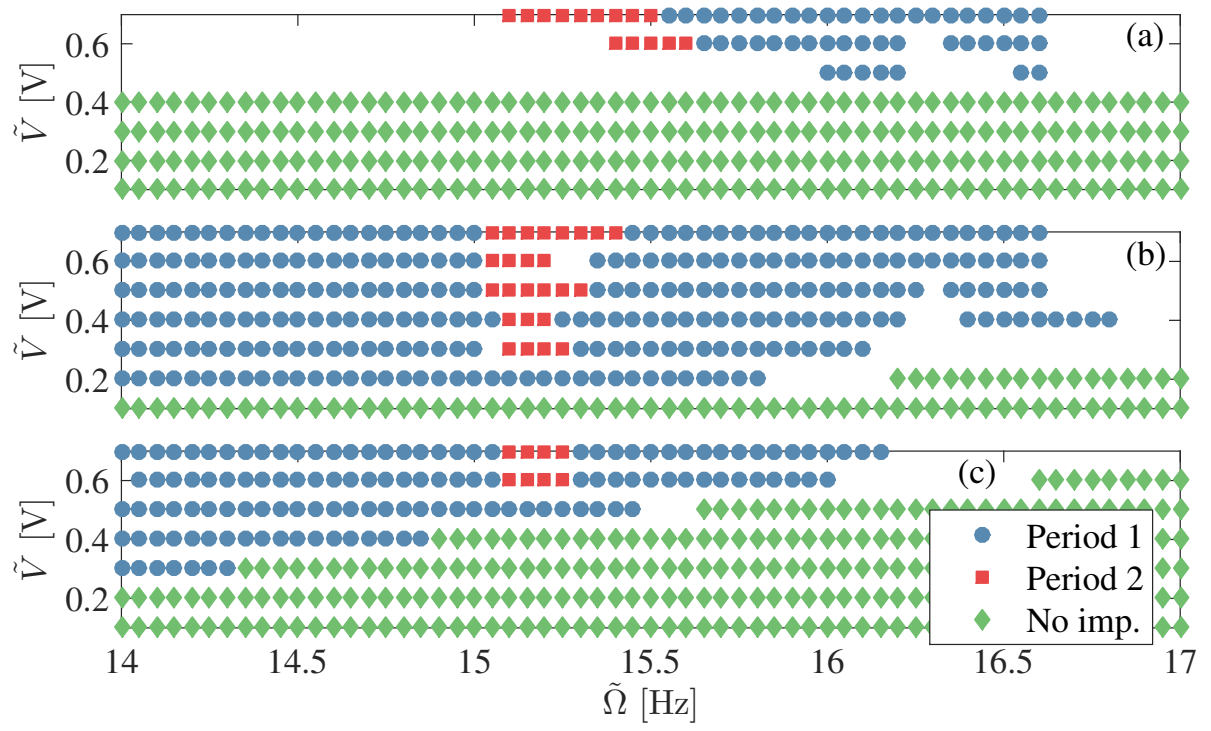

Figure 18: Periodicity for different gap configurations: (a) pre-stressed $-0.1 \mathrm{~mm}$, (b) neutral $0 \mathrm{~mm}$ and (c) loose $0.1 \mathrm{~mm}$.

The experimental frequency response for period one orbits can be seen in Fig. 19 , where the different excitation amplitudes and gap configurations are organized as fol- 
lows: excitation amplitude from $0.2 \mathrm{~V}$ to $0.4 \mathrm{~V}$ can be seen in Fig. 19 (c,e), while excitation amplitude from $0.5 \mathrm{~V}$ to $0.7 \mathrm{~V}$ can be seen in Fig. 19(a,b,d). The tight configuration with $\Delta_{G} \approx-0.1 \mathrm{~mm}$ can be seen in Fig. 19.a), the neutral configuration with $\Delta_{G} \approx 0 \mathrm{~mm}$ can be seen in Fig. 19 (b,c), and the loose configuration with $\Delta_{G} \approx 0.1 \mathrm{~mm}$ can be seen in Fig. 19(d,e). In this figure, it is not possible to identify an amplitude pattern for tightened configuration, shown in Fig. 19.a). The frequency response for neutral configuration Fig. 19 (b,c) is similar to the linear frequency response curve of SDOF systems with resonance around $15.5 \mathrm{~Hz}$ to $15.6 \mathrm{~Hz}$. For loose configuration Fig. 19. d,e), the amplitude is almost constant between $14 \mathrm{~Hz}$ to $15 \mathrm{~Hz}$, after that, it decreases, passing by a state of complex oscillations until there are no more vibroimpact oscillations.
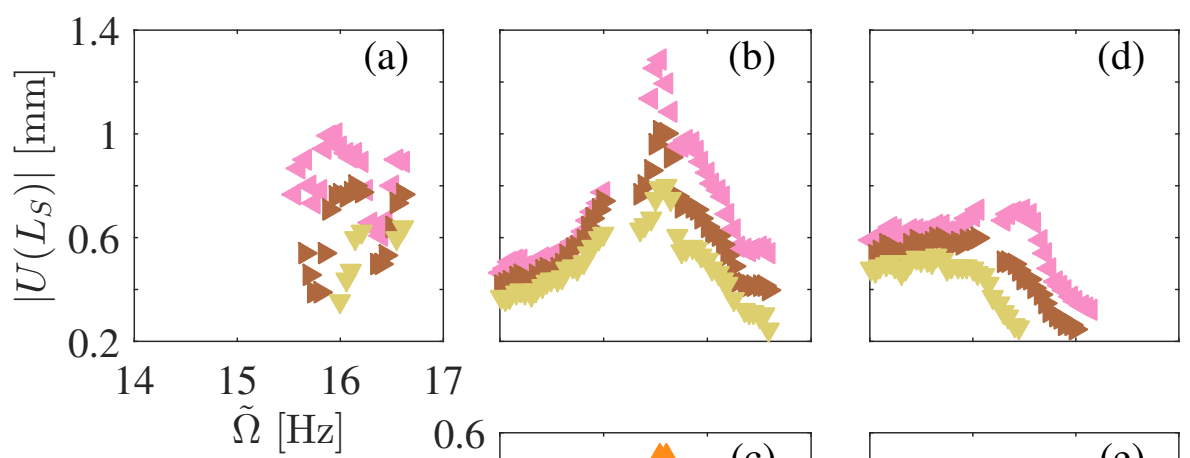

\begin{tabular}{|c|c|}
\hline$>$ & $0.7 \mathrm{~V}$ \\
$\nabla$ & $0.6 \mathrm{~V}$ \\
$\nabla$ & $0.5 \mathrm{~V}$ \\
$\triangle$ & $0.4 \mathrm{~V}$ \\
$\nabla$ & $0.3 \mathrm{~V}$ \\
& $0.2 \mathrm{~V}$ \\
\hline
\end{tabular}
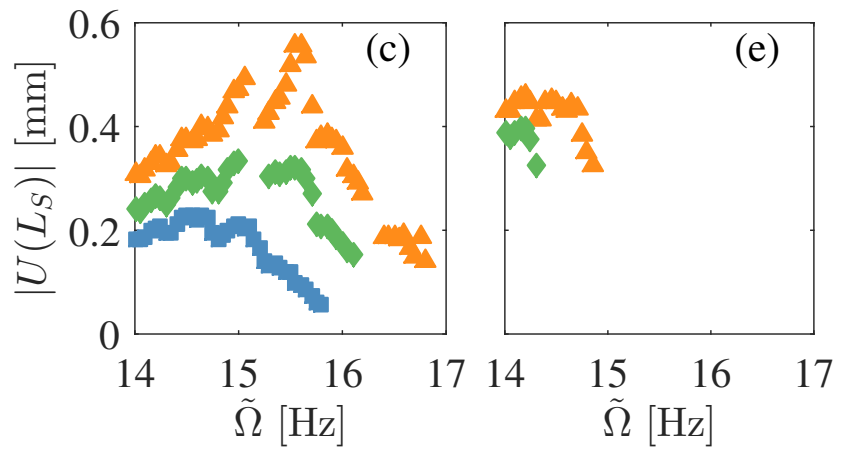

Figure 19: Experimental frequency response for different excitation levels $0.2 \mathrm{~V}$ to $0.7 \mathrm{~V}$ and gap configurations: (a) pre-stressed $-0.1 \mathrm{~mm},(\mathrm{~b}, \mathrm{c})$ neutral $0 \mathrm{~mm}$ and $(\mathrm{d}, \mathrm{e})$ loose $0.1 \mathrm{~mm}$.

The harmonic decomposition of period one orbits is shown in Fig. 20 a) to (c), for tight, neutral and loose configurations, respectively. There one can see that the fundamental harmonic $1 \tilde{\Omega}$ is the one with higher amplitude. The static component $0 \tilde{\Omega}$ also has high amplitude because the oscillations are asymmetric with respect to $U=0$. For neutral configuration Fig. 20 (b) one can see resonance-like curves for $0 \Omega \tilde{\Omega}$ and $1 \tilde{\Omega}$ with a peak close to $2 f_{n}$.

The different gap values should be understood as nominal measures because intermittent impacts during a frequency sweep and wear on the contacting surfaces can modify the initial gap width. Although not well studied, this is very common in engineering practice. This variation according to the forcing frequency is shown in Fig. 21, 

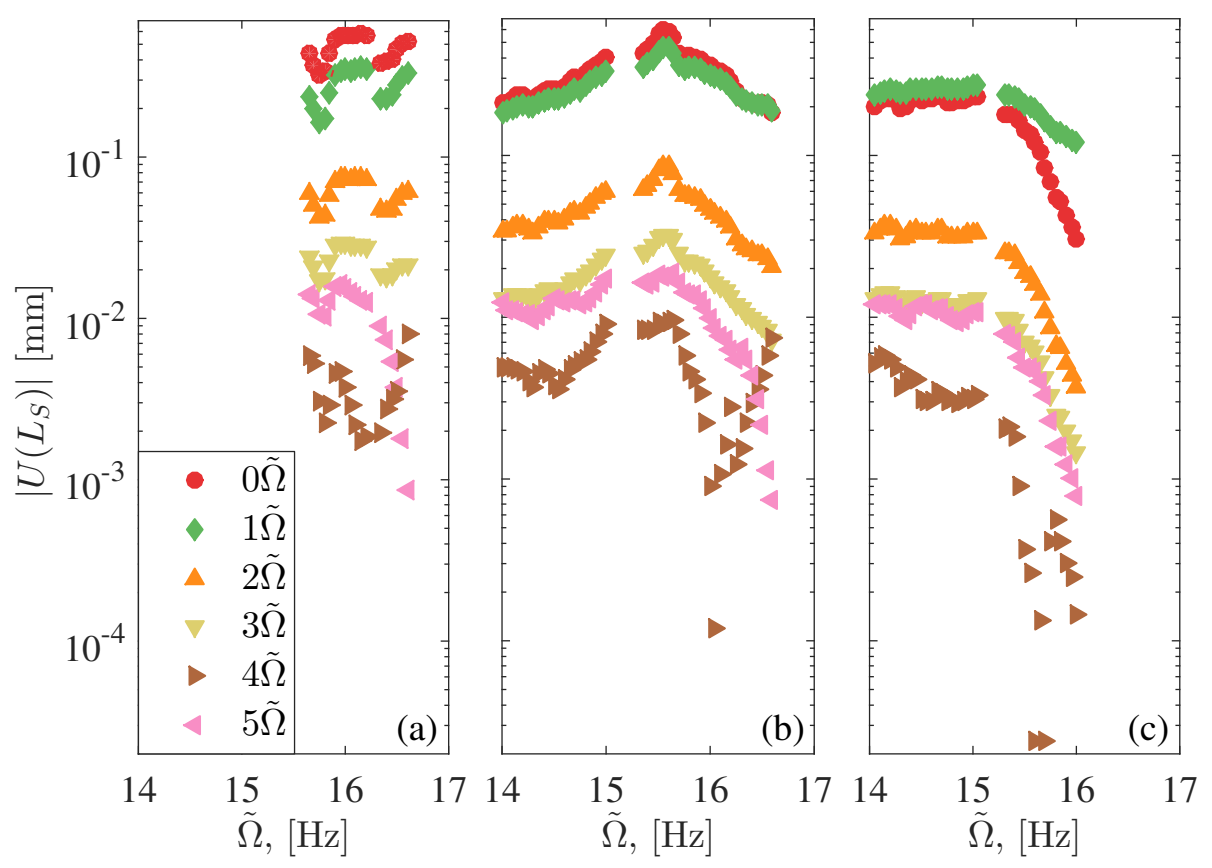

Figure 20: Harmonic decomposition of period one orbits for $\tilde{V}=0.6 \mathrm{~V}$ and different gap configurations: (a) pre-stressed $-0.1 \mathrm{~mm}$, (b) neutral $0 \mathrm{~mm}$ and (c) loose $0.1 \mathrm{~mm}$.

where the mass amplitude and measured gap width are shown in Fig. 21(a, b), (c, d) and (e, f) for tight, neutral and loose gap configurations, respectively. In this figure, it is possible to correlate some gap variations with the frequency response, such as the pattern similarity for $\left|U\left(L_{S}\right)\right|$ and $\Delta_{G}$ around $16 \mathrm{~Hz}$ on Fig. 21 (a, b) and the discontinuity of the FRC and the sudden increase of $\Delta_{G}$ around $15 \mathrm{~Hz}$ on Fig.21.c, d). The actual gap width was measured as the maximum value of vibrations for impacting orbits.

The results from measurements made at the neutral configuration and excitation level $0.6 \mathrm{~V}$ can be seen in Figs. 22 and 23 showing the different dynamic behaviors in the neighborhood of a period-doubling bifurcation. The stroboscopic map $S_{T}$ has one fixed point before bifurcation and two after that. Chaotic behavior is observed in the experimental data shown in Fig. 24 for pre-stressed conditions and $0.6 \mathrm{~V}$. In this case the stroboscopic map has no equilibrium points. The velocity was obtained from the displacement data using central finite differences.

It is not possible to ensure that the contact location will remain unchanged during the tests with different gap configurations, due to imperfections on the setup assembling, and on the surfaces of both the attached mass and constraint. Rotational vibrations of the attached rigid mass can occur due to eccentric collisions between the constraint and the mass, which has a significant moment of inertia. However, the amplitudes of the corresponding higher modes are generally much smaller than that of the beams first bending mode. The relevance of modal interactions for the present setup can be determined with an analysis of its vibrations in the frequency domain, as shown 

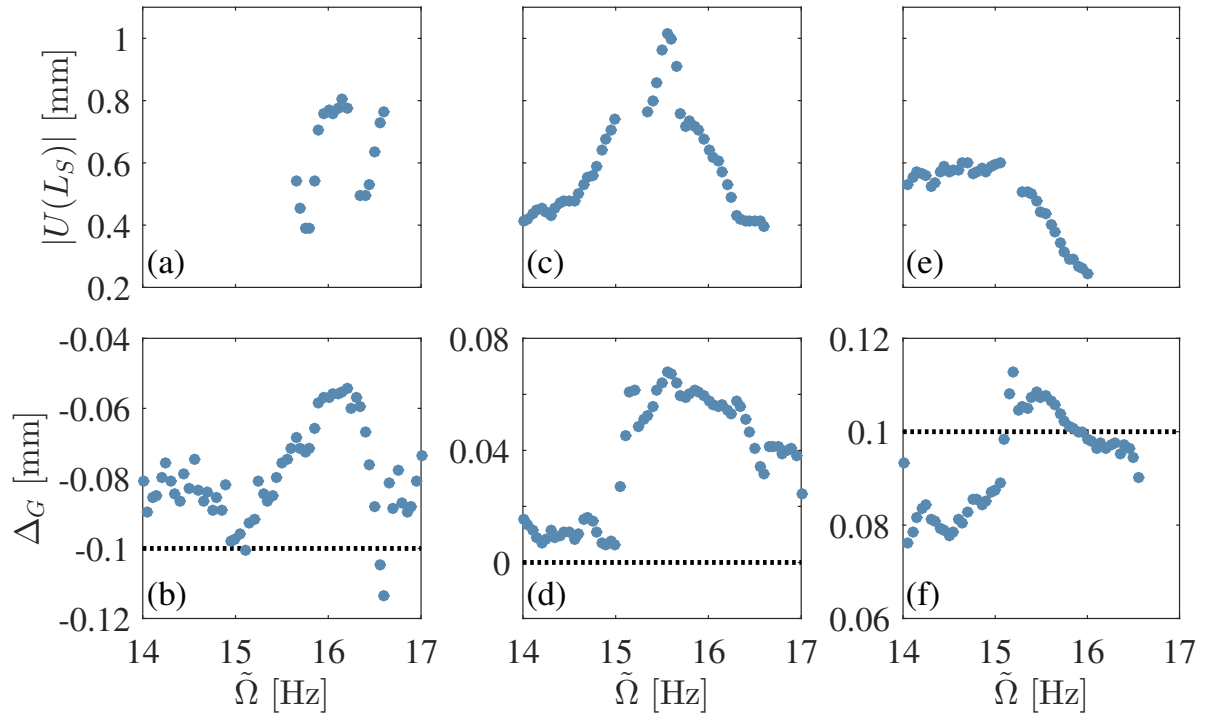

Figure 21: Period-1 frequency response (a, c, e) and gap variation (b, d, f). Excitation: 0.6 V.
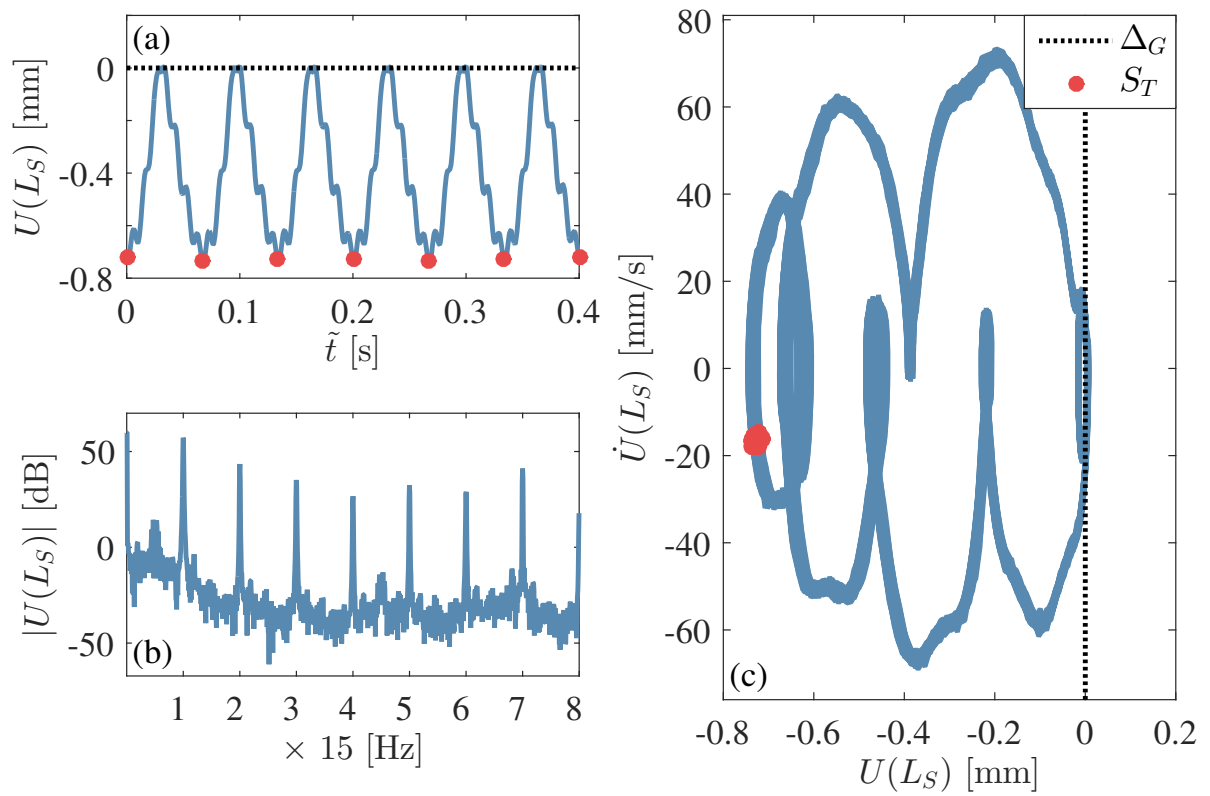

Figure 22: (a) Time series, (b) Frequency spectrum (c) phase-portrait.

471 in Figs. 20 and 22 to 24 , where only harmonics and sub-harmonics of the forcing fre472 quency can be identified. This indicates that the higher natural frequencies determined by experimental modal analysis do not seem to present a significant role for the vibro- 
474 impact response, thus justifying modeling the beam-mass setup as a SDOF system with 475 a point mass.
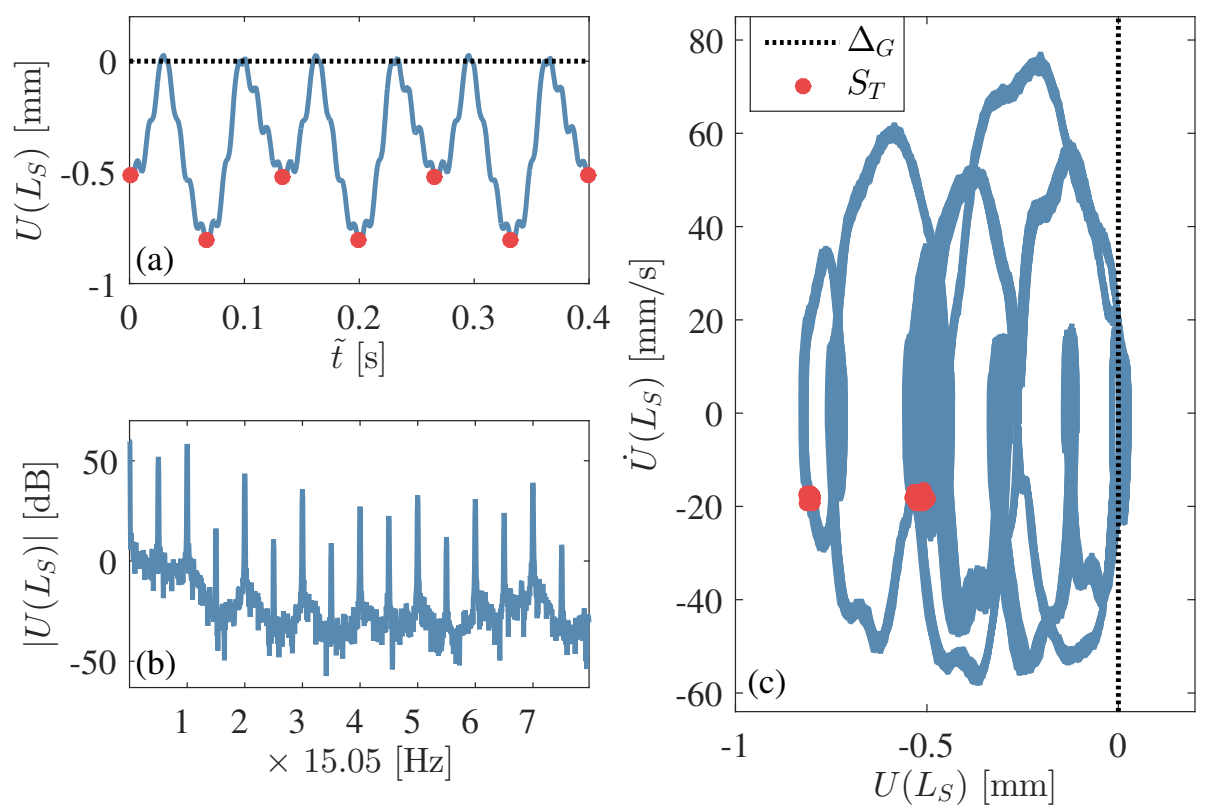

Figure 23: (a) Time series, (b) Frequency spectrum (c) phase-portrait. 

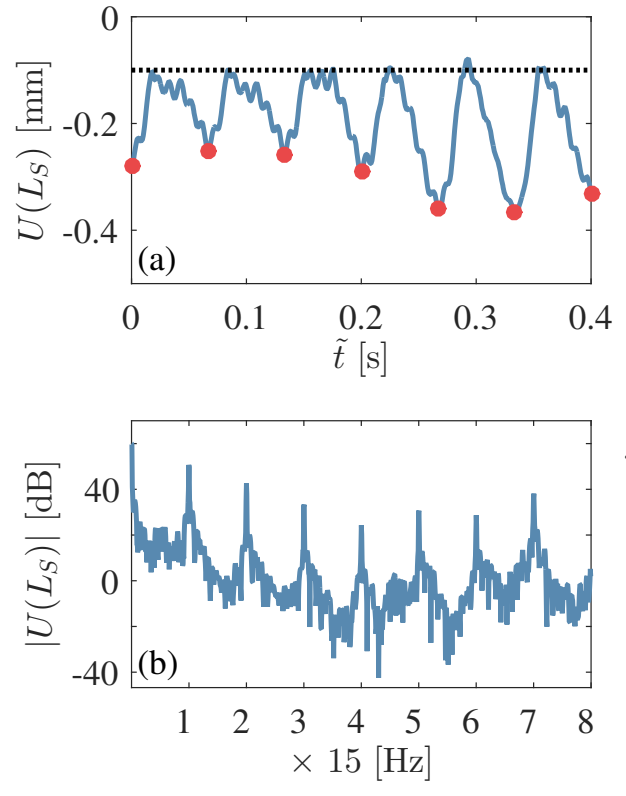

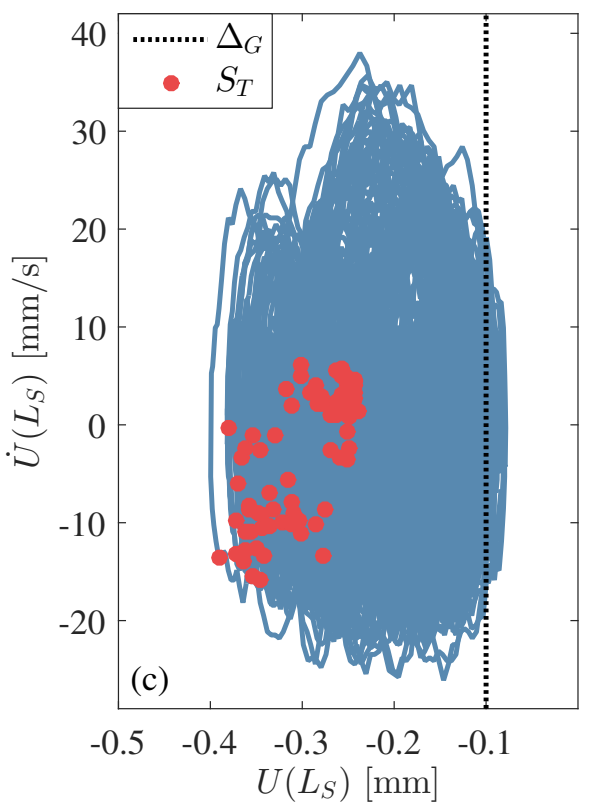

Figure 24: (a) Time series, (b) Frequency spectrum (c) phase-portrait.

\subsection{Coefficient of restitution}

The most common way of obtaining the coefficient of restitution is to pick the velocities before and after impact from an experimental time series and divide them $\left(R=-\dot{U}_{+} / \dot{U}_{-}\right)$. In general, the choice of $\dot{U}_{ \pm}$is made by picking up the initial and final points of intervals where velocity present an abrupt variation, changing its sign.

This approach was used on the first three impacts of period-one orbits, such as the one in Fig. 25, resulting in Fig. 26, which contains the value of $R$ for tight (a,b), neutral $(c, d)$ and loose (e, f) gap configurations as a function of forcing frequency (a, c, e) and pre-impact velocity $(\mathrm{b}, \mathrm{d}, \mathrm{f})$. In this figure, each dot represents the mean value of $R$ and/or $\dot{U}\left(L_{S}, \tilde{t}_{-}\right)$for the three impacts at each forcing frequency.

In Fig. 26(a) one can see that $R$ presents a piecewise linear growing pattern with the forcing frequency around $16 \mathrm{~Hz}$ and $16.5 \mathrm{~Hz}$. Similar behavior can be seen in Fig. 26. (c) for $R$ around $15.5 \mathrm{~Hz}$. Also, $R$ presents a decreasing pattern for $\dot{U}_{-}$from 0 to $30 \mathrm{~mm} \mathrm{~s}^{-1}$, see Fig. 26.d, f), which turns into a growing pattern for higher $\dot{U}_{-}$, see Fig. 26.d). The mean and standard deviation of $R$ for each gap are shown in Table 1 .

While the forcing frequency is a pre-defined parameter, the pre-contact velocity is influenced by both forcing frequency and gap configuration. That explains the dependence of the coefficient of restitution to the gap width shown in Table 1 where the mean value of $R$ decrease as its standard deviation grows together with the gap width. Alternatively, the gap dependence could indicate that the contact deformations are not negligible, invalidating the assumption used to model impacts with the CoR. That is not the case because contact deformations can be considered negligible if compared to the vibration amplitude, see Figs. 22 to 25 

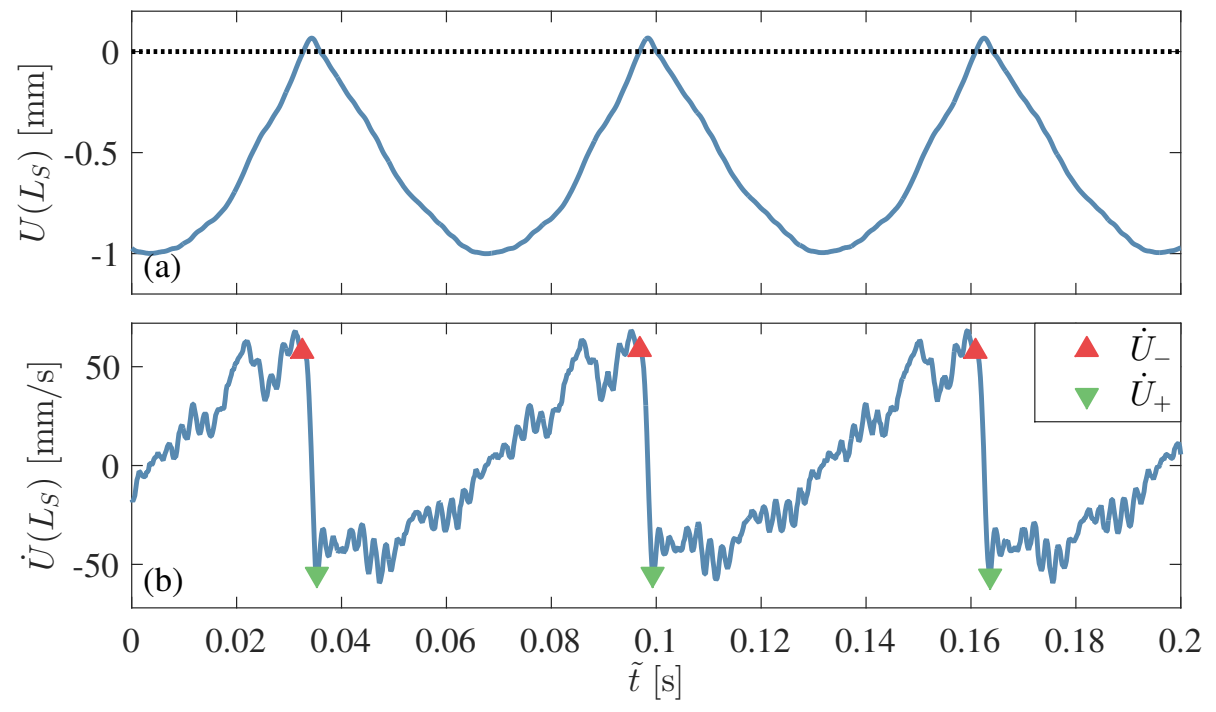

Figure 25: Displacement (a) and velocity (b) for $\Delta_{G} \approx 0 \mathrm{~mm}, \tilde{V}=0.6 \mathrm{~V}$ at $\tilde{\Omega}=15.6 \mathrm{~Hz}$.
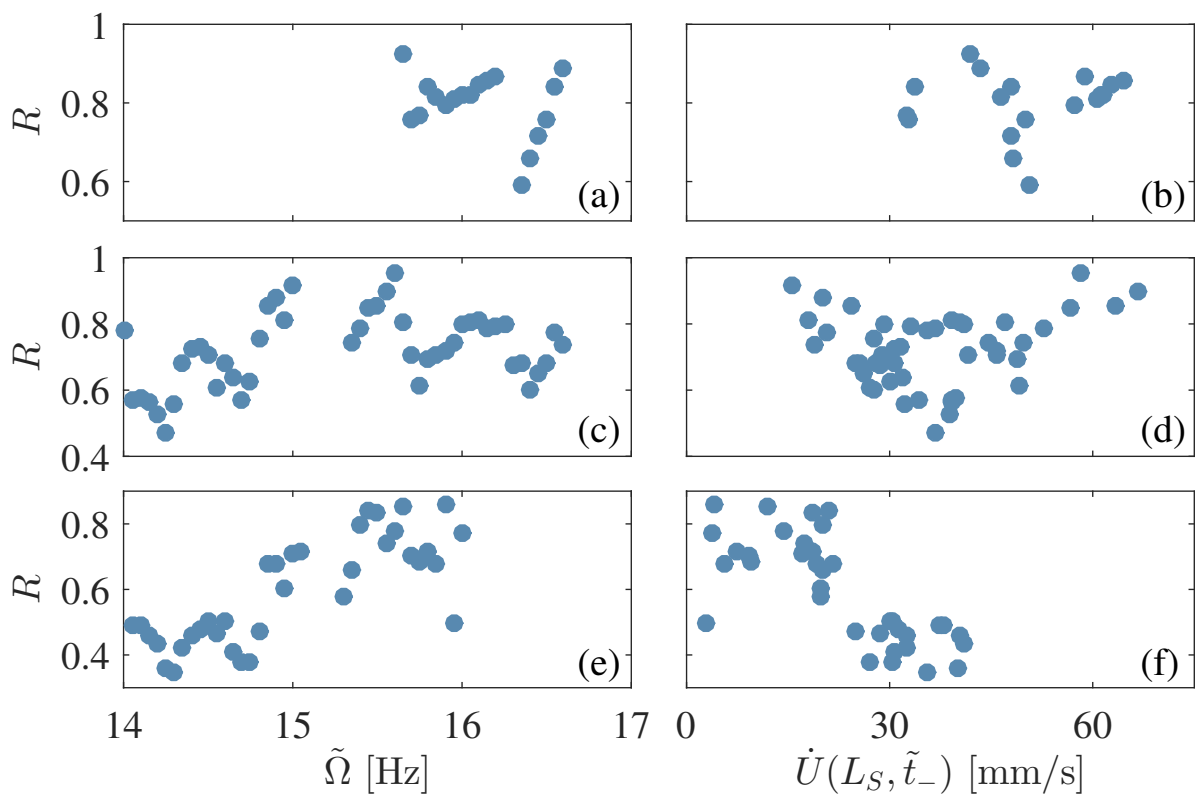

Figure 26: Coefficient of restitution for tight $\Delta_{G} \approx-0.1 \mathrm{~mm}(\mathrm{a}, \mathrm{b})$, neutral $\Delta_{G} \approx 0 \mathrm{~mm}(\mathrm{c}, \mathrm{d})$ and loose $\Delta_{G} \approx 0.1 \mathrm{~mm}(\mathrm{e}, \mathrm{f})$ gap configurations as a function of forcing frequency (a, c, e) and pre-impact velocity (b, d, f). $\tilde{V}=0.6 \mathrm{~V}$.

The uncertainty level of the coefficient of restitution and pre-impact velocity is 500 influenced by various factors, namely: 
Table 1: Coefficient of restitution against gap width

\begin{tabular}{ccc}
\hline \multirow{2}{*}{$\Delta_{G}[\mathrm{~mm}]$} & \multicolumn{2}{c}{$R$} \\
\cline { 2 - 3 } & mean & std. dev. \\
\hline-0.1 & 0.80 & 0.08 \\
0 & 0.72 & 0.11 \\
+0.1 & 0.59 & 0.16 \\
\hline
\end{tabular}

- Deviations on the gap width during operation, see Fig. 10

- Inherent mounting errors make it difficult to ensure identical impact conditions when the constraint location is changed;

- The velocity is not measured directly, being obtained through central finite differences from noise-contaminated position measurements;

- The pre- and post-contact velocities are chosen by visual observation of the time series, a task that can be difficult due to the presence of high-frequency components (HFC) as shown in Fig. 27 for the velocity;

Observing the velocity's frequency spectrum, shown in Fig. 27.b), only harmonics of the forcing frequency have noticeable amplitude, while the setup's resonances $(8 \mathrm{~Hz}$, $67 \mathrm{~Hz}$, and $98 \mathrm{~Hz}$ ) obtained in Section 5 are not prominent.

However, it is possible to observe the velocity's HFC in the time domain, see Fig. 27(c). Removing the low-frequency component of the velocity between impacts and using the average period between peaks, one obtains the signal's frequency as $103 \mathrm{~Hz}$. Looking at the velocity frequency spectrum in Fig. 27(b) it is possible to observe a small peak at this frequency. It is also possible to notice a small peak after $100 \mathrm{~Hz}$ in Fig. 17, that shows the results of the modal analysis. The low-frequency velocity in Fig. 27. a) was obtained through the optimization of the numeric response to match experimental data. The time series and frequency spectrum for the displacement associated with the velocity data shown in Fig. 27 can be seen in Fig. 22.

One can estimate the vibration amplitude of the HFC by considering that the velocity's amplitude is equal to the displacement amplitude times its oscillation frequency. The mean peak velocity amplitude of the HFC is $35 \mathrm{~mm} \mathrm{~s}^{-1}$ and its frequency is approximately $103 \mathrm{~Hz}$, resulting in a displacement amplitude of $0.05 \mathrm{~mm}$, which is more than 10 times smaller than the low-frequency displacement amplitude. Also, this component is not activated in the same way during the whole frequency and gap width domains, as one can see in Fig. 25.(b). 

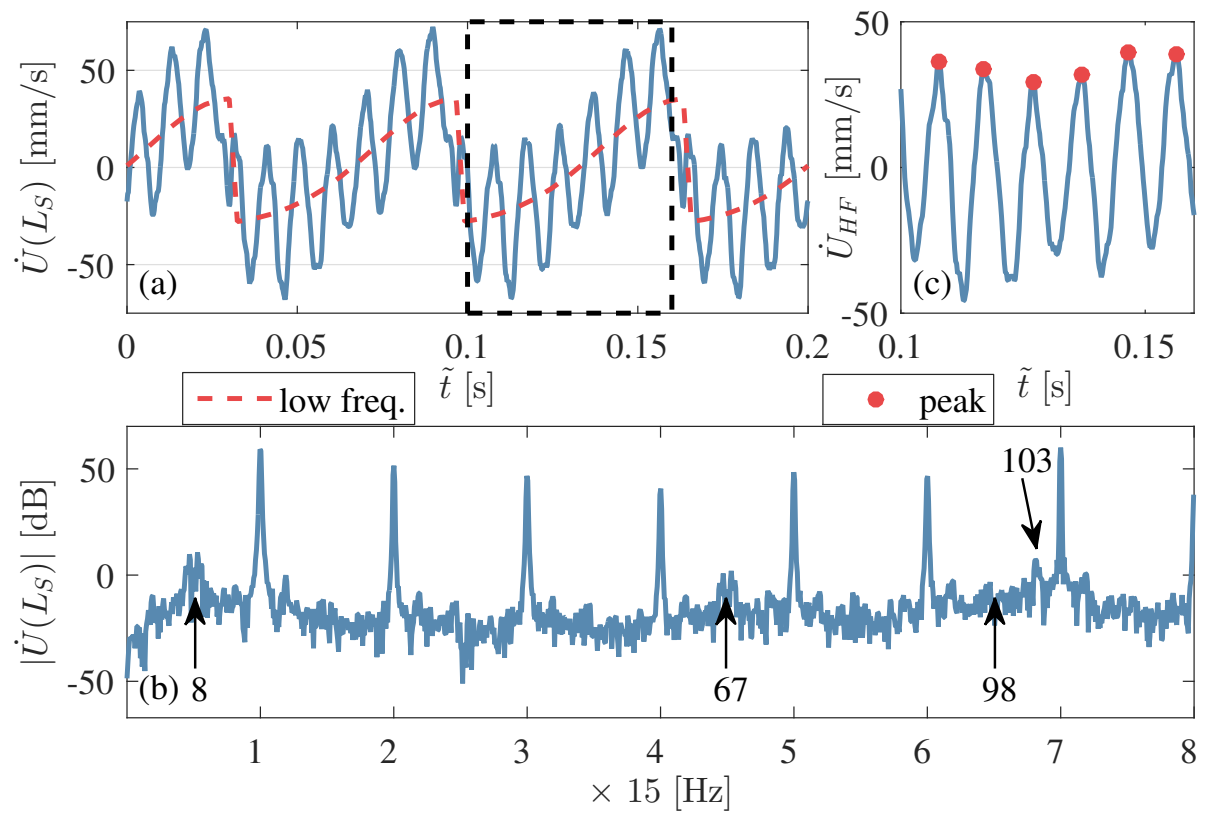

Figure 27: (a) Velocity time series for $\Delta_{G} \approx 0 \mathrm{~mm}, \tilde{V}=0.6 \mathrm{~V}, \tilde{\Omega}=15 \mathrm{~Hz}$. (b) Frequency spectrum for velocity. (c) High-frequency component of velocity at the boxed region shown in (a).

\subsection{Platform dynamics}

The influence of the impacting dynamics on the platform can be identified by comparing the frequency spectrum of colliding mass and platform shown in Fig. 28(c, d). Also, the platform acts as a low-pass filter on the excitation provided by the shaker, delivering reduced stimulation to the beam, as shown in Fig. 29, where its frequency response between $6 \mathrm{~Hz}$ and $9 \mathrm{~Hz}$ is at a higher level than at $14 \mathrm{~Hz}$ and $17 \mathrm{~Hz}$. Its variation between $7.5 \mathrm{~Hz}$ and $8 \mathrm{~Hz}$ is due to beam's resonance. This happens because the electrodynamic shaker is not feedback controlled. Thus, the chosen system's external excitation source is the single harmonic electric signal sent to the shaker's power amplifier. From that, one can write the system's platform displacement excitation as:

$$
b(\tilde{t})=\tilde{\gamma} \tilde{V} \sin (\tilde{\Omega} \tilde{t}),
$$

where $\tilde{V}$ is the voltage amplitude in $\mathrm{V}$ and $\tilde{\gamma}$ is the unit conversion gain in $\mathrm{m} \mathrm{V}^{-1}$.

The presence of a high-frequency component in the experimental data, together with the attenuating characteristics of the platform can be seen as motivation to update the mathematical model, adding more degrees of freedom, however, that would also make the use of techniques such as averaging and pointwise mapping impractical. Since the high-frequency component $(103 \mathrm{~Hz})$ is higher than the second and third beam's resonances $67 \mathrm{~Hz}$ and $98 \mathrm{~Hz}$, the inclusion of their corresponding modes on the model is not well-supported. Finally, incorporating the platform dynamics does not directly affect the impact behavior. 

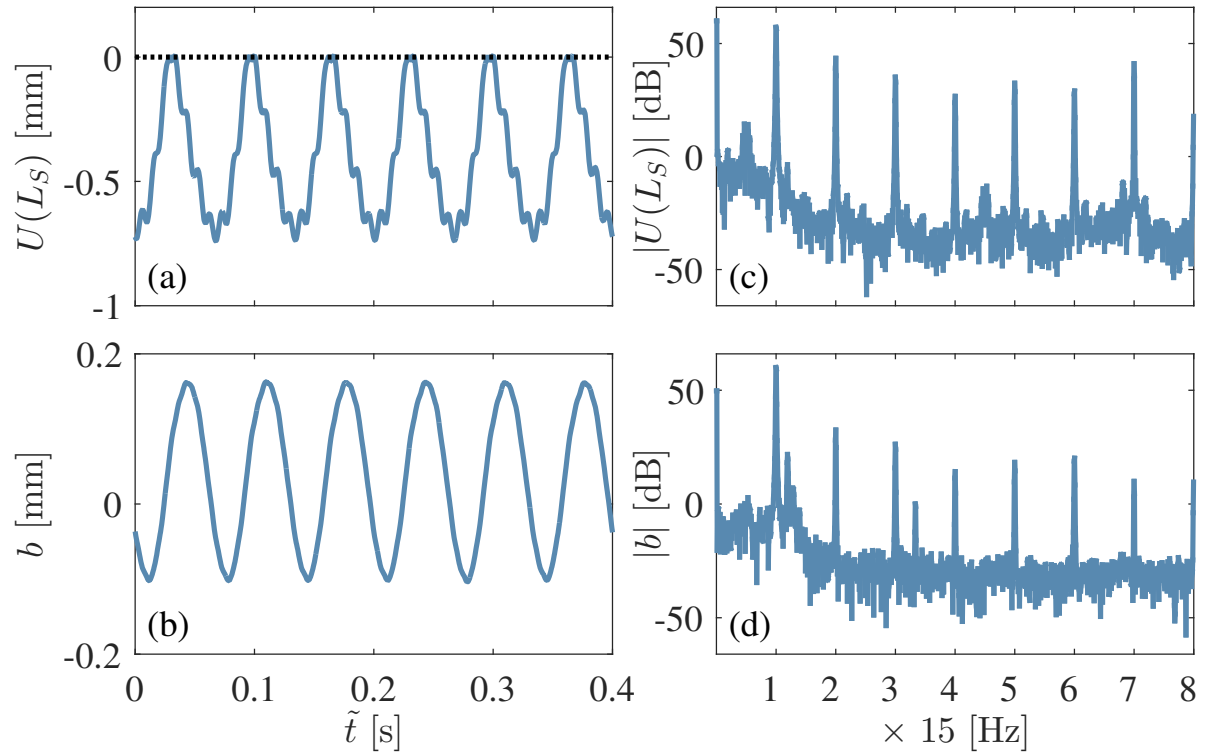

Figure 28: Displacement and frequency spectrum of (a,c) attached mass and (b,d) platform. $\Delta_{G} \approx 0.0 \mathrm{~mm}$, $0.6 \mathrm{~V}, \tilde{\Omega}=15 \mathrm{~Hz}$.

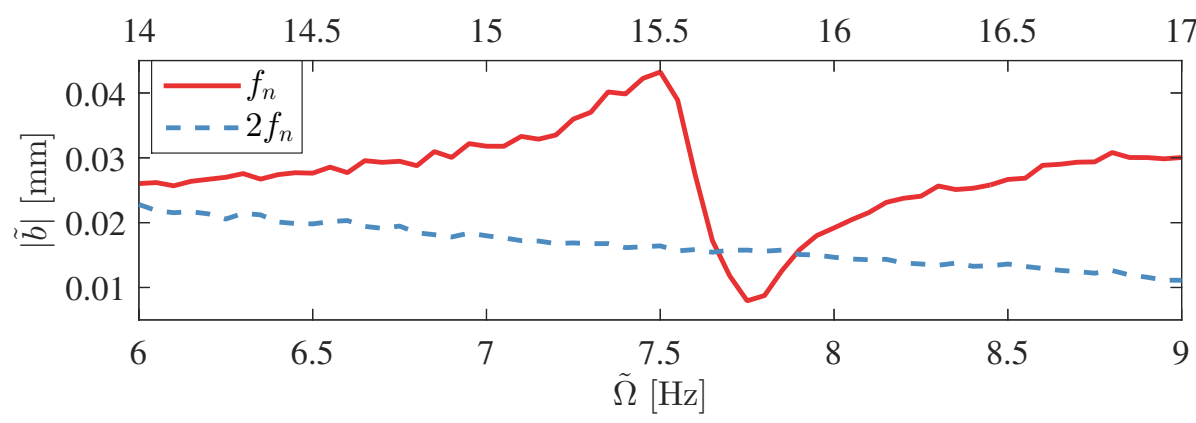

Figure 29: Platform response for $\tilde{V}=0.1 \mathrm{~V}$ around $1 \times$ and $2 \times$ resonance $f_{n}=7.6 \mathrm{~Hz}$.

\section{Model for the experimental setup}

The setup's model is equivalent to the one presented in Section 2, making it possible to investigate the occurrence of the predictions made in Sections 3 and 4 in experiments. Also, it is similar to the ones proposed by Elmegård et al. [45] and Rebouças et al. [62]. In [45] the impact was modeled using a piecewise relation for the spatial discretization of the equations of motion, while [62] modeled the impact as a force, normalizing the transverse displacement $U(X, \tilde{t})$ with the grazing amplitude $\Delta_{G}$. This would be a problem for the current analysis, which considers single-sided impacts whose gap can be null, leading to infinite normalized transverse vibrations. To avoid that, the beam length $L$ was used as a normalizing parameter. 
In the schematic representation of the forced impacting beam in Fig. [15 $, L_{m}, L_{S}$ and, $L$ represent the axial location of the attached mass, displacement sensor, and beam length, respectively, $\Delta_{C}$ represents the gap width. The stop has the same axial location of the attached mass, being placed on its right side. Using Bernoulli-Euler beam theory, one can write its equation of motion for transverse displacement $U(X, \tilde{t})$ as [45, 62]:

$$
\begin{array}{r}
\left(\rho A+m \delta\left(X-L_{m}\right)\right) \ddot{U}+D E I \dot{U}^{\prime \prime \prime \prime}+E I U^{\prime \prime \prime \prime}= \\
\left(\rho A+m \delta\left(X-L_{m}\right)\right) \tilde{\Omega}^{2} \gamma \tilde{V} \sin (\tilde{\Omega} \tilde{t}), \quad \text { if } \quad U_{m}<\Delta_{C}, \\
U(0, \tilde{t})=U^{\prime}(0, \tilde{t})=U^{\prime \prime}(L, \tilde{t})=U^{\prime \prime \prime}(L, \tilde{t})=0,
\end{array}
$$

where $(\cdot) \equiv \partial(\cdot) / \partial \tilde{t},(\cdot)^{\prime} \equiv \partial(\cdot) / \partial X, \rho, D, E, A$ and $I$ represent the beam's density, damping proportional to stiffness, elasticity modulus, cross-sectional area, and crosssection area moment of inertia, respectively, $m$ is the attached mass, $\delta(\cdot)$ is Dirac's delta function and $U_{m}=U\left(L_{m}, \tilde{t}\right)$ is the transverse displacement of the attached mass. Impact occurs when the mass reaches the stop, in which case:

$$
U\left(L_{m}, \tilde{t}_{-}\right)=\Delta_{C} \Rightarrow\left\{\begin{array}{l}
U\left(L_{m}, \tilde{t}_{+}\right)=U\left(L_{m}, \tilde{t}_{-}\right), \\
\dot{U}\left(L_{m}, \tilde{t}_{+}\right)=-R \dot{U}\left(L_{m}, \tilde{t}_{-}\right)
\end{array}\right.
$$

where $R$ is the coefficient of restitution, $\tilde{t}_{-}$and $\tilde{t}_{+}$represent instants before and after impact, respectively. Following the same steps taken in [62], namely:

- Normalize transversal displacement and axial position: $U=u L$ and $X=x L$;

- Assume that the attached mass is much larger than the beams: $m \gg \rho A L$;

- Perform spatial discretization:

$$
u(x, \tilde{t})=\sum_{i=1}^{N} \phi_{i}(x) q_{i}(\tilde{t}),
$$

where the assumed modes $\phi_{i}(x)$ satisfy all essential boundary conditions and $q_{i}(\tilde{t})$ are modal coordinates;

- Neglect higher modes: $N=1$;

One arrives at:

$$
\ddot{q}+2 \beta_{S} \dot{q}+q=\gamma \tilde{V} \Omega^{2} \sin (\Omega t), \quad \text { if } \quad q<\Delta,
$$

where:

$$
\begin{array}{r}
\beta_{S}=\frac{1}{2} D \omega_{n}, \quad \Omega=\tilde{\Omega} / \omega_{n}, \quad t=\omega_{n} \tilde{t}, \\
\Delta=\Delta_{G} / L, \quad \omega_{n}^{2}=K_{S} / M_{S}, \quad M_{S}=m \phi\left(x_{m}\right)^{2}, \\
K_{S}=\frac{E I}{L^{3}} \int_{0}^{1}\left(\phi^{\prime \prime}(x)\right)^{2} \mathrm{~d} x, \quad \gamma=\frac{\tilde{\gamma}}{\phi\left(x_{m}\right) L},
\end{array}
$$


are the structural damping ratio, normalized forcing frequency, normalized time, normalized gap width, squared fundamental linear natural frequency, equivalent structural mass, equivalent structural stiffness and normalized unit conversion gain. The assumed mode $\phi(\cdot)$ is chosen as the static deformation pattern of a cantilever beam loaded at $L_{m}$, normalized such that $\phi\left(L_{S}\right)=1$. Also, from now on $(\cdot) \equiv \partial(\cdot) / \partial t$. Applying the same steps to the impact condition, Eq. (2), one arrives at:

$$
\dot{q}\left(t_{+}\right)=-R \dot{q}\left(t_{-}\right) \quad \text { and } \quad q\left(t_{+}\right)=q\left(t_{-}\right) \quad \text { if } \quad q\left(t_{-}\right)=\Delta,
$$

where $t_{+}$and $t_{-}$are the normalized instants after and before impact, respectively.

Vibro-impact oscillations measured from the experimental setup can be related to Eq. (43) by multiplying the model solution by the measured grazing amplitude and by the first mode shape at the measurement location, $U\left(L_{S}, \widetilde{t}\right)=q\left(\omega_{n} \tilde{t}\right) L \phi\left(L_{S} / L\right)$. This relation can be simplified by normalizing the static deformation pattern $\phi(\cdot)$ with the measurement location, $L_{S}$. By doing so, the measured vibrations become proportional to the beam's length, i.e., $U\left(L_{S}, \tilde{t}\right)=q\left(\omega_{n} \tilde{t}\right) L$.

\subsection{Linear parameter estimation}

The system's structural damping ratio obtained through the decay envelope of the unforced SDOF response, such as the one shown in Fig. 30.a) together with its model prediction, indicating good agreement between them. So, from now on $\beta_{S}=17 \times 10^{-3}$. The frequency spectrum of the unforced response, shown in Fig. 30 (b) gives $f_{n}=7.75 \mathrm{~Hz}$, while the frequency sweep around this value, as shown in Fig. 30.(c) indicates that $f_{n}=7.6 \mathrm{~Hz}$, which is the value of the system's fundamental natural frequency from now on.

The normalized unit conversion gain $\gamma=1.5 \times 10^{-3} \mathrm{~V}^{-1}$ was obtained by fitting the experimental data shown in Fig. 30 (c) around $f_{n}$ to its theoretical estimative obtained with Eq. 43. Given the attenuating characteristic of the platform, c.f. Section 5.2, it may be necessary to modify this parameter during vibro-impact analysis (around $2 f_{n}$ ). 

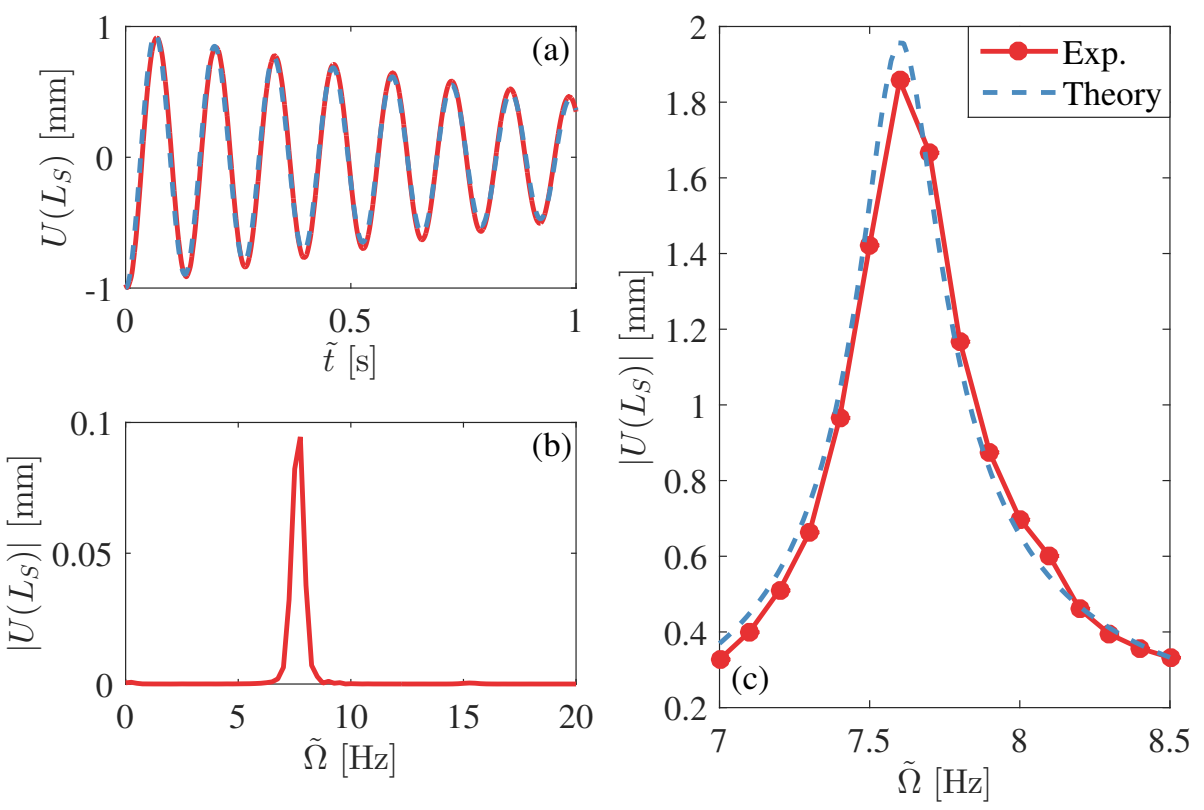

Figure 30: Linear transient response in (a) time and (b) frequency domains and (c) forced response in the frequency domain around fundamental resonance.

\section{Comparing Experimental and Numerical results}

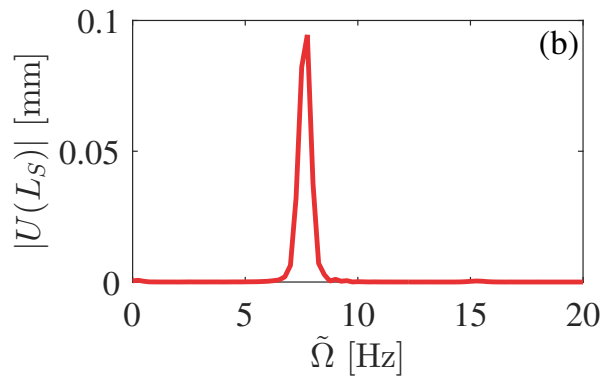

Remembering the attenuating characteristic of the platform, one can find a new value for $\gamma$ by fitting the frequency-amplitude obtained by numerical simulation to the experimental data shown previously. Using the mean values of $R$ for each gap width, given in Table 1 , one obtains the results shown in Fig. 31 with $\gamma=3.78 \times 10^{-4}$, $5.16 \times 10^{-4}$ and $7.66 \times 10^{-4}$ respectively, which corresponds to 25,34 and $51 \%$ of the initial value, obtained in Section 6.1 for measurements around resonance.

On tightened configuration Fig. 31(a), the experimental amplitude does not have a clear pattern, making it difficult to associate with numerical results. At neutral configuration Fig. 31 b), the numerical results present a reasonable agreement with the experimental ones. For loose configuration Fig. 31(c), the numerical frequency response is close to the experimental one for $\Omega \geq 2$, being able to predict the end of period-1 orbits before $\Omega=2.2$, and the beginning of non-periodic behavior. 

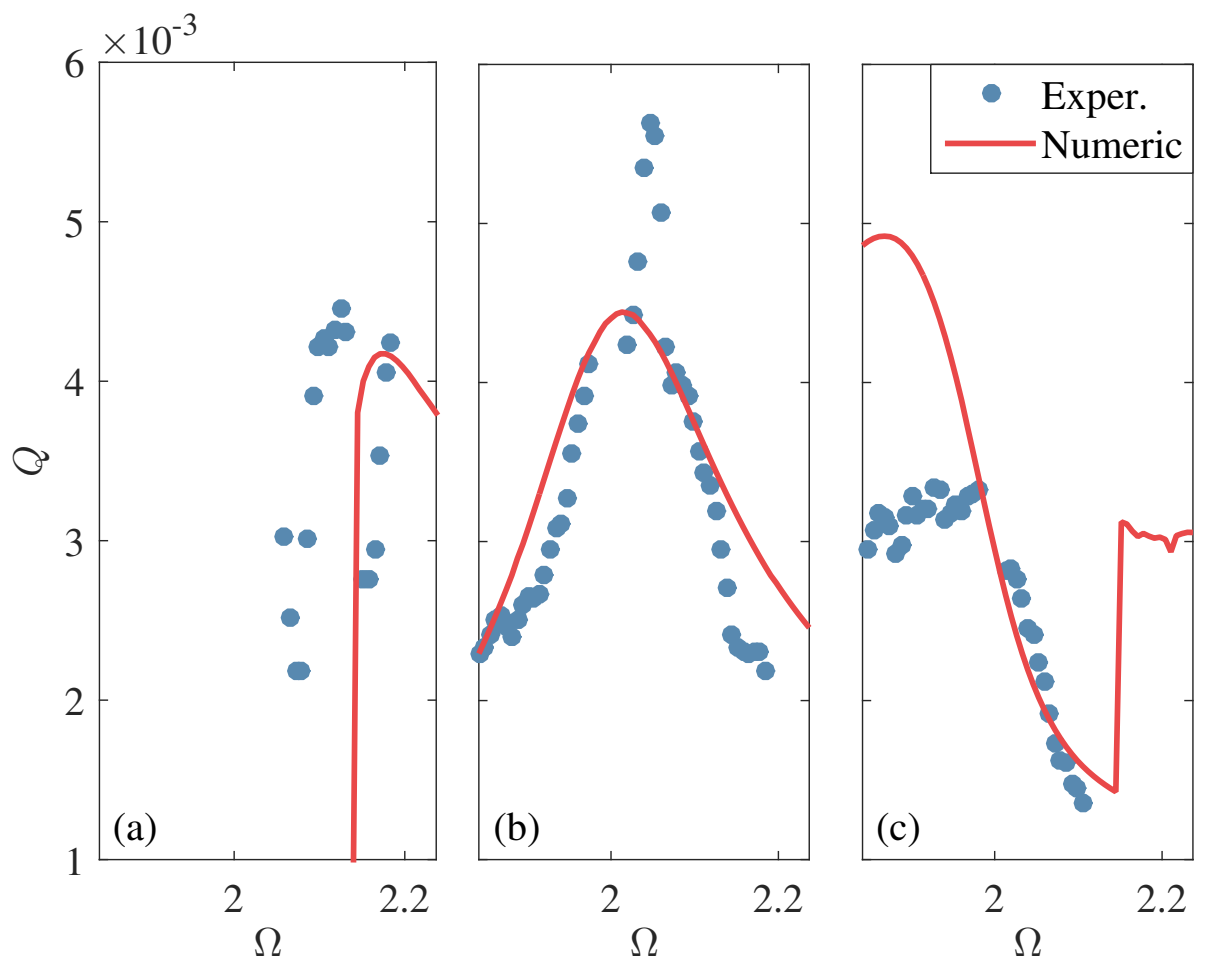

Figure 31: Experimental and numerical frequency responses. $\tilde{V}=0.6 \mathrm{~V}$. (a) $\Delta_{G} \approx-0.1 \mathrm{~mm}$, (b) $\Delta_{G} \approx 0 \mathrm{~mm}$ and (c) $\Delta_{G} \approx 0.1 \mathrm{~mm} . R$ is given by Table 1

\section{Conclusions}

The experimental vibro-impact behavior of a cantilever beam with unilateral impacts and base-excitation was presented considering different excitation levels and gap configurations (tight, neutral and loose). Analysis of the experimental data showed different qualitative behaviors near vibro-impact resonance and the effect of gap variation during a frequency sweep on the setup's amplitude response. Since the experiment's parameter identification and analysis were made in different frequency ranges, it was possible to show the challenges involved in tuning its vibro-impact model. The use of non-smooth transformations on numerical simulations and analytical schemes was discussed, showing the differences between Zhuravlev and Ivanov's substitutions and among averaging and pointwise mapping.

It was possible to obtain an agreement between numerical and experimental results for some gap configurations and frequency ranges. In the time domain, numerical and analytical predictions were able to match experimental data despite the occasional presence of high-frequency oscillations. Investigations on the coefficient of restitution $R$ showed its dependence on the pre-impact velocity and forcing frequency, presenting a linear behavior at some ranges.

Motivated by the work of Grace et al. [46], Zhuravlev and Ivanov transformations 
were used as pre- and post-processing steps on the numerical simulation of a model for a SDOF oscillator with unilateral impacts and coefficient of restitution. Comparison with direct numerical simulation showed that those transformations can decrease computation time without compromising the results. Ivanov's approach provided the smallest simulation time because it eliminated the impact condition. Results obtained with Zhuravlev's coordinates were different from the direct simulation for the velocity variable during impacts. When compared to Ivanov, Zhuravlev's transformation has a clear physical interpretation, with a straightforward derivation.

Stability boundaries in terms of the amplitude of oscillations and gap width were obtained through averaging analysis of the Zhuravlev transformed SDOF model. Interpretation of parametric relations from perturbation methods can be useful in the analysis of empirical systems that do not satisfy some of the theoretical assumptions. Despite the mismatch between numerical and experimental results showed in this work, the concentration of period- 1 orbits according to the gap sign (before/after impact resonance if the gap is positive/negative) agreed with predictions made by Zhuravlev [37] using averaging analysis for a SDOF model.

The frequency responses obtained with pointwise mapping and numerical simulation showed an unstable region for period-1 orbits which was not identified by the averaging analysis. With pointwise mapping, it was possible to obtain two stability equations, one for generic orbits and a simplified one for period- $n$ behavior. Both formulas were able to identify the instability threshold, with the general formula being able to identify more unstable orbits than its simplified version. However, both methods could not identify instability at some frequencies.

The experimental analysis carried out in this manuscript could be improved by finer tuning of the gap width, followed by a further empirical exploration of the vibro-impact behavior in tightened configuration. A feedback controlled shaker would diminish the concerns created by the attenuating characteristics of the platform. Alternatively, one could propose another model for the excitation source and/or an appropriate way to insert the high-frequency component on the model. Further contributions can be made through the application of the methods presented here for systems with bilateral impacts and rigid stops. In this case, the response magnitude is limited by the clearance width, and the response's frequency becomes the output of interest.

This work presents a structured discussion of common techniques for analysis of unilateral vibro-impact problems, discussing the common analytical and numerical schemes. Despite experimental challenges, using the coefficient of restitution it was possible to reproduce empirical observations to a certain extent, validating analytical predictions.

\section{Acknowledgments}

The authors express their acknowledgment to CAPES through the Science Without Borders Program with the process number: 99999.013736/2013-02, which sponsored the elaboration of this paper. 


\section{References}

[1] S. W. Shaw, P. J. Holmes, A periodically forced piecewise linear oscillator, Journal of Sound and Vibration 90 (1) (1983) 129-155. doi:10.1016/ 0022-460X(83) 90407-8.

[2] F. C. Moon, S. W. Shaw, Chaotic vibrations of a beam with non-linear boundary conditions, International Journal of Non-Linear Mechanics 18 (6) (1983) 465477. doi:10.1016/0020-7462(83) 90033-1.

[3] S. W. Shaw, Forced vibrations of a beam with one-sided amplitude constraint: Theory and experiment, Journal of Sound and Vibration 99 (2) (1985) 199-212. doi:10.1016/0022-460x(85) 90357-8.

[4] S. Lin, C. Bapat, Extension of Clearance and Impact Force Estimation Approaches to a Beam-Stop System, Journal of Sound and Vibration 163 (3) (1993) 423-428. doi:10.1006/jsvi.1993.1179.

[5] G. Blankenship, A. Kahraman, Steady state forced response of a mechanical oscillator with combined parametric excitation and clearance type non-linearity, Journal of Sound and Vibration 185 (5) (1995) 743-765. doi:10.1006/ jsvi.1995.0416.

[6] S. Aoki, T. Watanabe, Forced Vibration of Continuous System with Unsymmetrical Collision Characteristics, Nonlinear Dynamics 17 (2) (1998) 141-157. doi:10.1023/A:1008207721738.

[7] A. C. J. Luo, The mapping dynamics of periodic motions for a three-piecewise linear system under a periodic excitation, Journal of Sound and Vibration 283 (35) (2005) 723-748. doi:10.1016/j.jsv.2004.05.023.

[8] U. Andreaus, L. Placidi, G. Rega, Soft impact dynamics of a cantilever beam: equivalent SDOF model versus infinite-dimensional system, Proceedings of the Institution of Mechanical Engineers, Part C: Journal of Mechanical Engineering Science 225 (10) (2011) 2444-2456. doi:10.1177/0954406211414484.

[9] V. R. Hiwarkar, V. I. Babitsky, V. V. Silberschmidt, On the modelling of dynamic structures with discontinuities, Nonlinear Dynamics 67 (4) (2012) 2651-2669. doi:10.1007/s11071-011-0178-0.

[10] B. Uspensky, K. Avramov, Nonlinear modes of piecewise linear systems under the action of periodic excitation, Nonlinear Dynamics 76 (2) (2014) 1151-1156. doi:10.1007/s11071-013-1198-8.

[11] A. J. Dick, B. Balachandran, H. Yabuno, M. Numatsu, K. Hayashi, M. Kuroda, K. Ashida, Utilizing nonlinear phenomena to locate grazing in the constrained motion of a cantilever beam, Nonlinear Dynamics 57 (3) (2009) 335-349. doi : 10.1007/s11071-008-9445-0. 
[12] R. R. Aguiar, H. I. Weber, Mathematical modeling and experimental investigation of an embedded vibro-impact system, Nonlinear Dynamics 65 (3) (2011) 317334. doi:10.1007/s11071-010-9894-0.

[13] U. Andreaus, P. Baragatti, L. Placidi, Experimental and numerical investigations of the responses of a cantilever beam possibly contacting a deformable and dissipative obstacle under harmonic excitation, International Journal of Non-Linear Mechanics 80 (2016) 96-106. doi:10.1016/j.ijnonlinmec.2015. 10.007 .

[14] S. Liu, Q. Cheng, D. Zhao, L. Feng, Theoretical modeling and analysis of twodegree-of-freedom piezoelectric energy harvester with stopper, Sensors and Actuators A: Physical 245 (2016) 97-105. doi:10.1016/j.sna.2016.04. 060 .

[15] K. H. Hunt, F. R. E. Crossley, Coefficient of Restitution Interpreted as Damping in Vibroimpact, Journal of Applied Mechanics 42 (2) (1975) 440. doi : $10.1115 /$ 1.3423596 .

[16] H. M. Lankarani, P. E. Nikravesh, A Contact Force Model With Hysteresis Damping for Impact Analysis of Multibody Systems, Journal of Mechanical Design 112 (3) (1990) 369. doi:10.1115/1.2912617.

[17] M. Machado, P. Moreira, P. Flores, H. M. Lankarani, Compliant contact force models in multibody dynamics: Evolution of the Hertz contact theory, Mechanism and Machine Theory 53 (2012) 99-121. doi:10.1016/j. mechmachtheory.2012.02.010.

[18] A. Stensson, A. B. Nordmark, Experimental Investigation of Some Consequences of Low Velocity Impacts in the Chaotic Dynamics of a Mechanical System, Philosophical Transactions of the Royal Society A: Mathematical, Physical and Engineering Sciences 347 (1683) (1994) 439-448. doi:10.1098/rsta.1994. 0053 .

[19] W. Fang, J. Wickert, Response of a Periodically Driven Impact Oscillator, Journal of Sound and Vibration 170 (3) (1994) 397-409. doi:10.1006/jsvi. 1994.1070.

[20] S. R. Bishop, M. G. Thompson, S. Foale, Prediction of period-1 impacts in a driven beam, Proceedings of the Royal Society of London A: Mathematical, Physical and Engineering Sciences 452 (1954) (1996) 2579-2592. doi: $10.1098 / \mathrm{rspa} .1996 .0137$.

[21] D. J. Wagg, S. R. Bishop, Application of non-smooth modelling techniques to the dynamics of a flexible impacting beam, Journal of Sound and Vibration 256 (5) (2002) 803-820. doi:10.1006/jsvi.2002.5020.

[22] R. Davis, L. Virgin, Non-linear behavior in a discretely forced oscillator, International Journal of Non-Linear Mechanics 42 (5) (2007) 744-753. doi: $10.1016 / j . i j n o n l i n m e c .2007 .02 .012$ 
[23] J. J. Thomsen, A. Fidlin, Near-elastic vibro-impact analysis by discontinuous transformations and averaging, Journal of Sound and Vibration 311 (1-2) (2008) 386-407. doi:10.1016/j.jsv.2007.09.007

[24] B. Blazejczyk-Okolewska, K. Czolczynski, T. Kapitaniak, Dynamics of a twodegree-of-freedom cantilever beam with impacts, Chaos, Solitons \& Fractals 40 (4) (2009) 1991-2006. doi:10.1016/j.chaos.2007.09.097

[25] C. P. Vyasarayani, J. McPhee, S. Birkett, Modeling Impacts Between a Continuous System and a Rigid Obstacle Using Coefficient of Restitution, Journal of Applied Mechanics 77 (2) (2010) 021008. doi:10.1115/1.3173667.

[26] O. V. Gendelman, Analytic treatment of a system with a vibro-impact nonlinear energy sink, Journal of Sound and Vibration 331 (21) (2012) 4599-4608. doi: $10.1016 / j \cdot j s v .2012 .05 .021$.

[27] H. Ruan, T. Yu, The unexpectedly small coefficient of restitution of a two-degreeof-freedom mass-spring system and its implications, International Journal of Impact Engineering 88 (2016) 1-11. doi:10.1016/j.ijimpeng.2015.09. 005 .

[28] P. Müller, M. Heckel, A. Sack, T. Pöschel, Complex Velocity Dependence of the Coefficient of Restitution of a Bouncing Ball, Physical Review Letters 110 (25) (2013) 254301. doi:10.1103/PhysRevLett.110.254301.

[29] B. Blazejczyk-Okolewska, K. Czolczynski, T. Kapitaniak, Hard versus soft impacts in oscillatory systems modeling, Communications in Nonlinear Science and Numerical Simulation 15 (5) (2010) 1358-1367. doi:10.1016/j.cnsns. 2009.05 .046 .

[30] A. E. Kobrinskii, Dynamics of mechanisms with elastic connections and impact systems, Iliffe, London, 1969.

[31] A. C. J. Luo, Y. Guo, Vibro-Impact Dynamics, John Wiley \& Sons Ltd, 2013. doi:10.1002/9781118402924.

[32] V. I. Babitsky, Theory of Vibro-Impact Systems and Applications, Foundations of Engineering Mechanics, Springer Berlin Heidelberg, Berlin, Heidelberg, 1998. doi:10.1007/978-3-540-69635-3.

[33] A. Narimani, Frequency Response of a Piecewise Linear Vibration Isolator, Journal of Vibration and Control 10 (12) (2004) 1775-1794. doi:10.1177/ 1077546304044795 .

[34] K. Zou, S. Nagarajaiah, Study of a piecewise linear dynamic system with negative and positive stiffness, Communications in Nonlinear Science and Numerical Simulation 22 (1-3) (2015) 1084-1101. doi:10.1016/j.cnsns. 2014 . 08.016 . 
[35] V. N. Pilipchuk, Nonlinear Dynamics, Vol. 52 of Lecture Notes in Applied and Computational Mechanics, Springer Berlin Heidelberg, Berlin, Heidelberg, 2010. doi:10.1007/978-3-642-12799-1.

[36] V. N. Pilipchuk, Analytical Study of Vibrating Systems With Strong Nonlinearities by Employing Saw-tooth Time Transformations, Journal of Sound and Vibration 192 (1) (1996) 43-64. doi:10.1006/jsvi.1996.0175.

[37] V. Zhuravlev, Equations of motion of mechanical systems with ideal onesided links, Journal of Applied Mathematics and Mechanics 42 (5) (1978) 839-847. doi:10.1016/0021-8928(78)90031-X.

[38] A. P. Ivanov, Analytical methods in the theory of vibro-impact systems, Journal of Applied Mathematics and Mechanics 57 (2) (1993) 221-236. doi:10.1016/ 0021-8928(93)90050-V.

[39] A. Fidlin, Nonlinear Oscillations in Mechanical Engineering, Springer-Verlag, Berlin/Heidelberg, 2006. doi:10.1007/3-540-28116-9.

[40] V. F. Zhuravlev, Method of Analysis of Vibration-Shock Systems by Means of Special Functions, Mechanics of Solids 11 (2) (1976) 30-34.

[41] V. N. Pilipchuk, Closed-form solutions for oscillators with inelastic impacts, Journal of Sound and Vibration 359 (2015) 154-167. doi:10.1016/j.jsv. 2015.08 .023 .

[42] K. Czolczynski, B. Blazejczyk-Okolewska, A. Okolewski, Analytical and numerical investigations of stable periodic solutions of the impacting oscillator with a moving base, International Journal of Mechanical Sciences 115-116 (2016) 325338. doi:10.1016/j.ijmecsci.2016.07.004.

[43] M. Wiercigroch, Modelling of dynamical systems with motion dependent discontinuities, Chaos, Solitons \& Fractals 11 (15) (2000) 2429-2442. doi: $10.1016 /$ S0960-0779(00)00032-1.

[44] M. A. Savi, S. Divenyi, L. F. P. Franca, H. I. Weber, Numerical and experimental investigations of the nonlinear dynamics and chaos in non-smooth systems, Journal of Sound and Vibration 301 (1-2) (2007) 59-73. doi:10.1016/j.jsv. 2006.09 .014 .

[45] M. Elmegård, B. Krauskopf, H. M. Osinga, J. Starke, J. J. Thomsen, Bifurcation analysis of a smoothed model of a forced impacting beam and comparison with an experiment, Nonlinear Dynamics 77 (3) (2014) 951-966. doi:10.1007/ s11071-014-1353-x

[46] I. M. Grace, R. A. Ibrahim, V. N. Pilipchuk, Inelastic impact dynamics of ships with one-sided barriers. Part I: analytical and numerical investigations, Nonlinear Dynamics 66 (4) (2011) 589-607. doi:10.1007/s11071-010-9937-6. 
[47] V. Acary, B. Brogliato, Numerical Methods for Nonsmooth Dynamical Systems, Vol. 35 of Lecture Notes in Applied and Computational Mechanics, Springer Berlin Heidelberg, Berlin, Heidelberg, 2008. doi:10.1007/ 978-3-540-75392-6.

[48] L. Dieci, L. Lopez, A survey of numerical methods for IVPs of ODEs with discontinuous right-hand side, Journal of Computational and Applied Mathematics 236 (16) (2012) 3967-3991. doi:10.1016/j.cam.2012.02.011.

[49] V. Acary, Projected event-capturing time-stepping schemes for nonsmooth mechanical systems with unilateral contact and Coulomb's friction, Computer Methods in Applied Mechanics and Engineering 256 (2013) 224-250. doi:10. $1016 / \mathrm{j} . \mathrm{cma} .2012 .12 .012$.

[50] B. Balachandran, Dynamics of an Elastic Structure Excited by Harmonic and 1. Aharmonic Impactor Motions, Modal Analysis 9 (3-4) (2003) 265-279. doi: $10.1177 / 107754603030752$.

[51] J. P. Cusumano, M. T. Sharkady, B. W. Kimble, Experimental Measurements of Dimensionality and Spatial Coherence in the Dynamics of a Flexible-Beam Impact Oscillator, Philosophical Transactions of the Royal Society A: Mathematical, Physical and Engineering Sciences 347 (1683) (1994) 421-438. doi : $10.1098 /$ rsta.1994.0052.

[52] M. A. Azeez, A. F. Vakakis, Proper Orthogonal Decomposition (POD) of a Class of Vibroimpact Oscillations, Journal of Sound and Vibration 240 (5) (2001) 859889. doi:10.1006/jsvi.2000.3264.

[53] K. Czolczynski, A. Okolewski, B. Blazejczyk-Okolewska, Lyapunov exponents in discrete modelling of a cantilever beam impacting on a moving base, International Journal of Non-Linear Mechanics 88 (2017) 74-84. doi:10.1016/J. I JNONLINMEC.2016.10.015,

[54] M. D. Todd, L. N. Virgin, An experimental impact oscillator, Chaos, Solitons \& Fractals 8 (4) (1997) 699-714. doi:10.1016/S0960-0779(96) $00112-9$

[55] M. Wiercigroch, V. W. T. Sin, Experimental Study of a Symmetrical Piecewise Base-Excited Oscillator, Journal of Applied Mechanics 65 (3) (1998) 657. doi: $10.1115 / 1.2789108$

[56] J. Ing, E. Pavlovskaia, M. Wiercigroch, S. Banerjee, Experimental study of impact oscillator with one-sided elastic constraint., Philosophical transactions. Series A, Mathematical, physical, and engineering sciences 366 (1866) (2008) 679-704. doi:10.1098/rsta.2007.2122.

[57] L. N. Virgin, C. George, A. Kini, Experiments on a non-smoothly-forced oscillator, Physica D: Nonlinear Phenomena 313 (2015) 1-10. doi:10.1016/j. physd.2015.09.002. 
[58] B. Brogliato, Viscoelastic Contact/Impact Rheological Models, in: Nonsmooth Mechanics, Springer, Cham, 2016, pp. 51-93. doi:10.1007/ 978-3-319-28664-8_2.

[59] R. A. Ibrahim, Vibro-Impact Dynamics, Vol. 43 of Lecture Notes in Applied and Computational Mechanics, Springer Berlin Heidelberg, Berlin, Heidelberg, 2009. doi:10.1007/978-3-642-00275-5.

[60] E. Bureau, F. Schilder, I. F. Santos, J. J. Thomsen, J. Starke, Experimental bifurcation analysis of an impact oscillator-Tuning a non-invasive control scheme, Journal of Sound and Vibration 332 (22) (2013) 5883-5897. doi:10.1016/ j.jsv.2013.05.033.

[61] E. Bureau, F. Schilder, M. Elmegård, I. F. Santos, J. J. Thomsen, J. Starke, Experimental bifurcation analysis of an impact oscillator-Determining stability, Journal of Sound and Vibration 333 (21) (2014) 5464-5474. doi:10.1016/ j.jsv.2014.05.032.

[62] G. F. S. Rebouças, I. F. Santos, J. J. Thomsen, Validation of vibro-impact force models by numerical simulation, perturbation methods and experiments, Journal of Sound and Vibration 413 (2018) 291-307. doi:10.1016/j.jsv.2017. 10.028 .

[63] S. W. Shaw, P. J. Holmes, A Periodically Forced Impact Oscillator With Large Dissipation, Journal of Applied Mechanics 50 (4a) (1983) 849. do i:10.1115/ 1.3167156. 